\title{
Initial Measurements with the Prototype Parallel-Slit Ring Collimator Fast Neutron Emission Tomography System
}

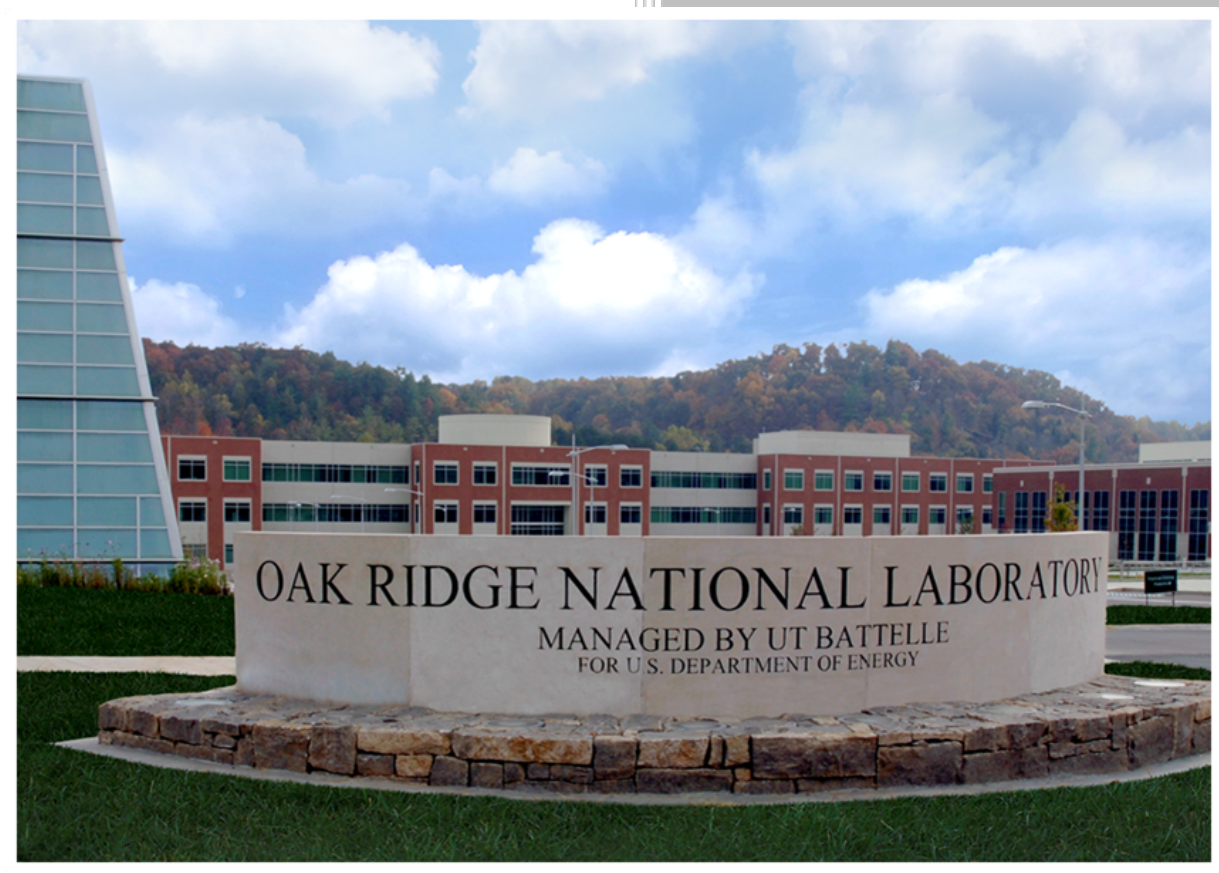

Approved for public release. Distribution is unlimited.

P. A. Hausladen

M. E. Montague

L. Fabris

August 2021 


\title{
DOCUMENT AVAILABILITY
}

Reports produced after January 1, 1996, are generally available free via US Department of Energy (DOE) SciTech Connect.

Website www.osti.gov

Reports produced before January 1, 1996, may be purchased by members of the public from the following source:

\author{
National Technical Information Service \\ 5285 Port Royal Road \\ Springfield, VA 22161 \\ Telephone 703-605-6000 (1-800-553-6847) \\ TDD 703-487-4639 \\ Fax 703-605-6900 \\ E-mail info@ntis.gov \\ Website http://classic.ntis.gov/
}

Reports are available to DOE employees, DOE contractors, Energy Technology Data Exchange representatives, and International Nuclear Information System representatives from the following source:

Office of Scientific and Technical Information

PO Box 62

Oak Ridge, TN 37831

Telephone 865-576-8401

Fax 865-576-5728

E-mail reports@osti.gov

Website http://www.osti.gov/contact.html

This report was prepared as an account of work sponsored by an agency of the United States Government. Neither the United States Government nor any agency thereof, nor any of their employees, makes any warranty, express or implied, or assumes any legal liability or responsibility for the accuracy, completeness, or usefulness of any information, apparatus, product, or process disclosed, or represents that its use would not infringe privately owned rights. Reference herein to any specific commercial product, process, or service by trade name, trademark, manufacturer, or otherwise, does not necessarily constitute or imply its endorsement, recommendation, or favoring by the United States Government or any agency thereof. The views and opinions of authors expressed herein do not necessarily state or reflect those of the United States Government or any agency thereof. 
Physics Division

INITIAL MEASUREMENTS WITH THE PROTOTYPE PARALLEL-SLIT RING COLLIMATOR FAST NEUTRON EMISSION TOMOGRAPHY SYSTEM

\author{
P. A. Hausladen \\ M. E. Montague \\ L. Fabris
}

August 2021

Prepared by

OAK RIDGE NATIONAL LABORATORY

Oak Ridge, TN 37831-6283

managed by

UT-BATTELLE, LLC

for the

US DEPARTMENT OF ENERGY

under contract DE-AC05-00OR22725 



\section{CONTENTS}

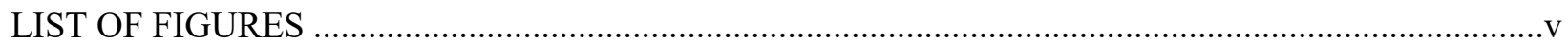

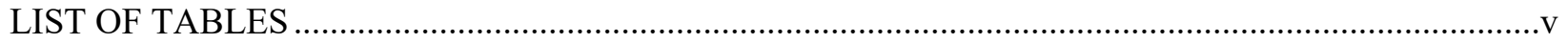

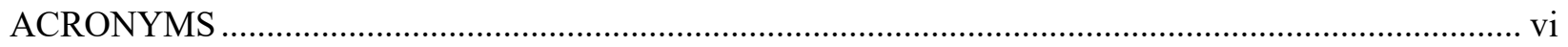

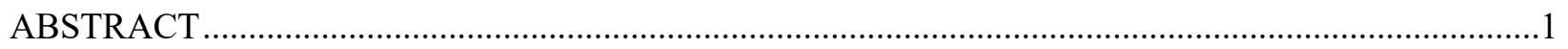

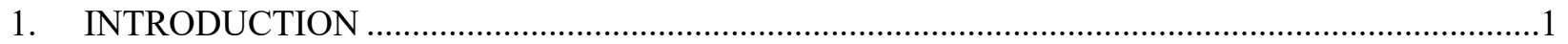

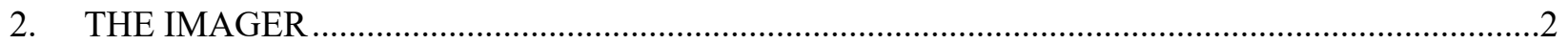

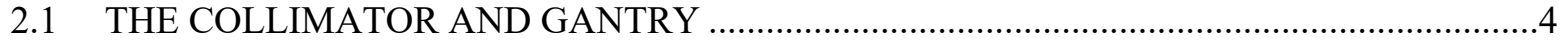

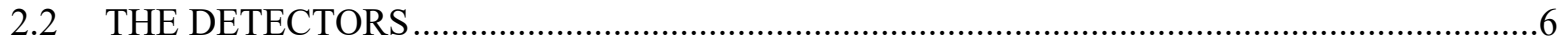

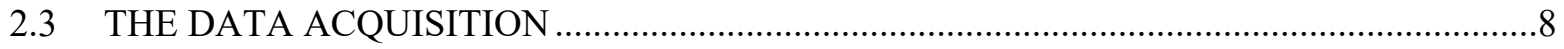

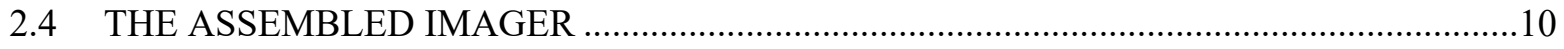

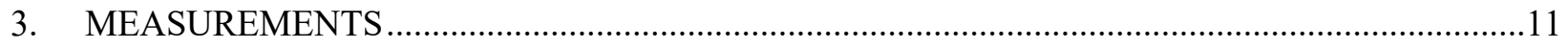

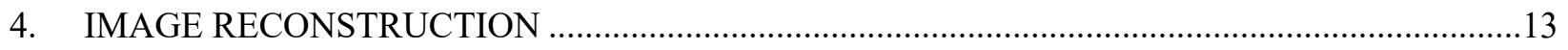

4.1 RECONSTRUCTION OF MEASUREMENTS OF INDIVIDUAL SOURCES ....................14

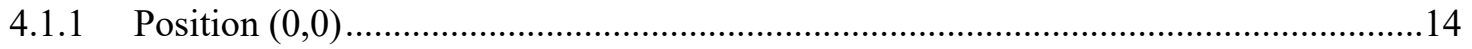

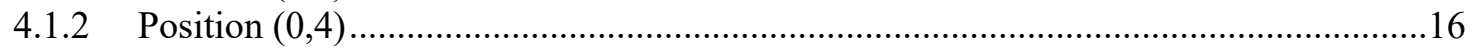

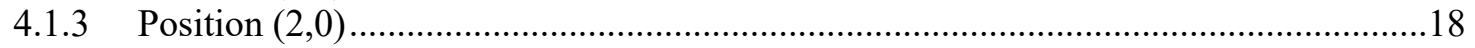

4.2 RECONSTRUCTION OF FIVE-SOURCE MEASUREMENT …........................................19

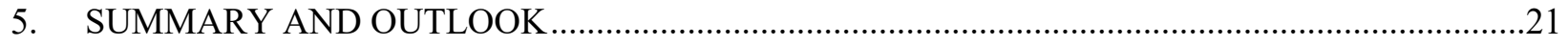

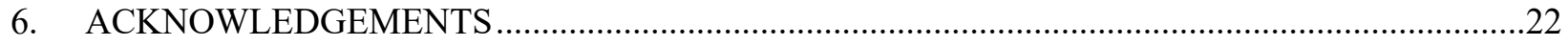

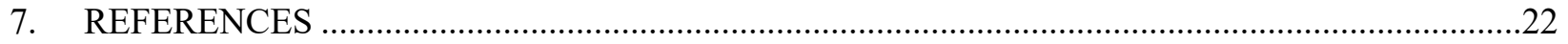





\section{LIST OF FIGURES}

Figure 1. Plan view of the imager design.

Figure 2. The collimator septa during assembly showing (a) the nickel-plated steel portions and

(b) the assembled composite steel and borated polyethylene septa.

Figure 3. Assembly of the collimator....

Figure 4. A detector module during assembly, including (a) a preamplifier board for a row of straws and a module with (b) the straw ends with grounding and connector sockets visible, (c) the 24 preamplifiers installed, (d) the high voltages and discriminator outputs connected, and (e) the module lid in place.

Figure 5. The set of 6 detectors (a) mounted with spacers to form a complete ring, and (b) with the addition of shielding, power, and logic signal cables during detector testing.

Figure 6. The data acquisition system.

Figure 7. CAEN A967 flat ribbon cable, "68-pin P50 connector to two $2.54 \mathrm{~mm} 34$ pin male connectors" (https://www.caen.it/products/a967/) ).................................................................

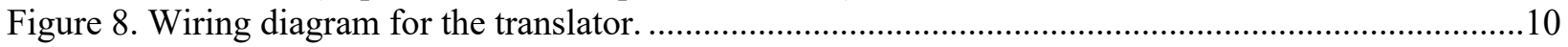

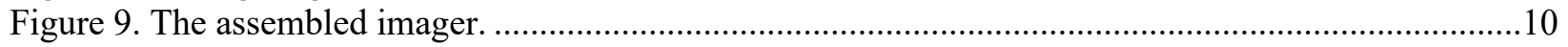

Figure 10. Fixture that mimics a $17 \times 17$ PWR nuclear fuel assembly .................................................11

Figure 11. Fixture that mimics $17 \times 17$ PWR nuclear fuel assembly..................................................12

Figure 12. Example projection for neutron source at position $(0,0)$ and collimator at initial

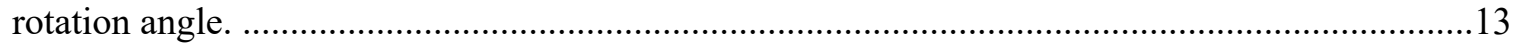

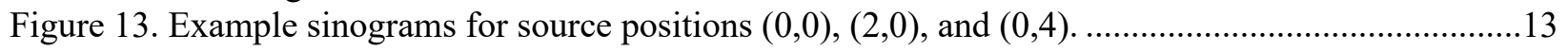

Figure 14. The (left) measured and (right) reconstructed sinogram for source position $(0,0) \ldots \ldots \ldots \ldots \ldots \ldots \ldots . . .14$

Figure 15. Measured data compared to reconstruction for position $(0,0)$, projections $10,20,30$,

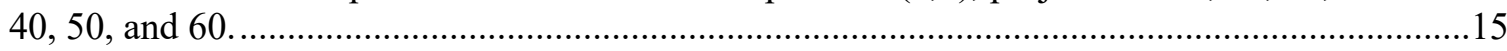

Figure 16. The reconstructed image for position $(0,0)$ for (left) the entire field of view and (right)

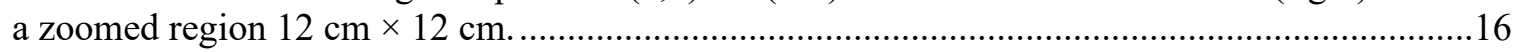

Figure 23. The (left) measured and (right) reconstructed sinograms for the sum of five positions.............20

Figure 24. Comparison of measurement and MLEM reconstruction for projections 10, 20, 30, 40,

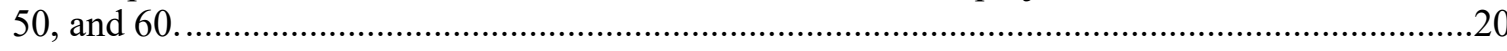

Figure 25. Image of five sources reconstructed using the MLEM algorithm for (left) 100

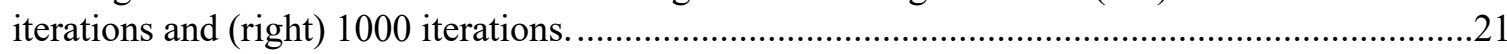

\section{LIST OF TABLES}

Table 1: Summary of initial measurements performed. 


\section{ACRONYMS}

$\begin{array}{ll}\text { DAQ } & \text { data acquisition } \\ \text { FOV } & \text { field of view } \\ \text { FWHM } & \text { full width at half maximum } \\ \text { HDPE } & \text { high-density polyethylene } \\ \text { LVDS } & \text { low voltage differential signaling } \\ \text { MLEM } & \text { maximum likelihood expectation maximization } \\ \text { ORNL } & \text { Oak Ridge National Laboratory } \\ \text { PTI } & \text { Proportional Technologies Inc. } \\ \text { VME } & \text { Versa Module Europa }\end{array}$





\begin{abstract}
Since 2017, Oak Ridge National Laboratory (ORNL) has been developing a passive fast-neutron emission tomography capability. The goal of this development is the ability to quantify the neutron source strength of individual fuel pins (rods) in spent nuclear fuel assemblies. Such a system could be used to measure the burnup of each fuel pin in a spent fuel assembly to take burnup credit when loading dry storage casks or to count individual fuel pins in spent fuel assemblies for safeguards purposes. At present, a laboratory prototype imager has been built and initial imaging measurements performed. The purpose of this prototype is to demonstrate imaging capability sufficient to resolve individual fuel pins in spent fuel assemblies, and in initial measurements, neutron sources separated by a spacing of $1.27 \mathrm{~cm}$ (similar to the spacing between fuel pins in commercial pressurized water reactor $17 \times 17$ nuclear fuel assemblies) have been resolved. This report documents the as-built imager, first measurements performed with it, and tomographic reconstructions performed using the measured data.
\end{abstract}

\title{
1. INTRODUCTION
}

ORNL is developing a new capability for passive fast-neutron emission tomography. The goal of this development is the ability to quantify the neutron source strength of individual fuel pins (rods) in spent nuclear fuel assemblies, the unit of special nuclear material in nuclear reactors. This technology could be used to measure the burnup of each fuel pin in a spent fuel assembly to take burnup credit for loading dry storage casks. This technology could also be used like the passive gamma emission tomography capability presently being evaluated by the International Atomic Energy Agency to detect the removal or substitution of individual fuel pins in spent nuclear fuel assemblies for international safeguards applications, such as to verify the integrity of an assembly before transfer to difficult-to-access storage [1-2].

Emission tomography using neutrons is largely unexplored. In practice, neutron emission tomography was not developed earlier because the scarcity of neutron-emitting materials (they are only made as byproducts of the nuclear fuel cycle) limits potential applications, and the modest intensities of typical neutron sources compared to the corresponding gamma ray intensities makes gamma measurements preferable for many applications. Moreover, the ability of fast neutrons to penetrate a good deal of shielding makes them difficult to collimate and measure with the required resolution. However, the use of fast neutrons to perform emission tomography of spent nuclear fuel is desirable for several reasons.

Neutrons readily penetrate the high-atomic-number fuel in the assemblies, making the contribution of neutrons from fuel pins from the interior of the assembly readily measurable. Neutrons originate from actinides and are more linked to the fissile material content of the fuel than gamma rays. Neutron measurements are more sensitive to assemblies containing fuel pins that were replaced after a single cycle in the reactor and subsequently irradiated in the core. This sensitivity is a consequence of the neutron signal originating primarily from the ingrowth of ${ }^{244} \mathrm{Cm}$, which occurs predominantly at the end of an exposure cycle. Also, neutron measurements are sufficiently sensitive that active measurements are possible and thereby provide a potential pathway to enable direct measurement of the fissile content of spent fuel, measurement of fuels with low burnup, and measurement of fuels with very long cooling times.

Despite the wide diagnostic use of $\mathrm{x}$-ray computed tomography and emission tomography modalities such as positron emission tomography and single photon emission computed tomography in medical physics, it is valuable to highlight aspects of computed tomography that relate to the working principles of the fastneutron imager. 
In each form of computed tomography, projection data from many angles are used to reconstruct crosssectional images of an inspected object for diagnostic purposes. The crucial elements of this process are that measured data divide the object into "lines of response," and multiple views through the object are mathematically combined to estimate an image. A line of response refers to a path through the inspected object along which an observable, such as neutron emission activity, can be integrated. For passive neutron imaging of a fuel assembly, a collimator slit defines the line of response through the fuel assembly, and neutron counts in a detector at the terminus of a collimator slit correspond primarily to an integral of neutron activity along the path defined by the slit. Fast-neutron tomography with the desired resolution is possible provided each line of response sufficiently isolates a path through the object, the object is sampled with sufficient lines of response, and there are sufficient views through the object to invert measured data to form an image. The resulting tomographic reconstruction shows an image of the neutron emission rate per pixel from which the emission rate from each fuel pin in the assembly can be extracted.

The combined challenges associated with effectively collimating neutrons, achieving sufficient resolution, maintaining sufficient efficiency for the modest neutron source strength from spent fuel, and maintaining sufficient insensitivity to the gamma-ray flux to allow meaningful neutron measurements were addressed by developing a new collimator concept termed a "parallel-slit ring collimator." The physics design of this imager was previously reported in [3-4, 6-8]. Development of the iterative reconstruction code used to perform tomographic reconstruction, the imager response calculation used by the reconstruction code, and the results of reconstructions of simulated tomographic imaging measurements for the prototype imager design were documented in [5].

At present, a laboratory prototype imager has been built and initial imaging measurements performed. The purpose of this prototype imager is to demonstrate sufficient capability to resolve individual fuel pins in a spent nuclear fuel assembly. This report documents initial measurements that demonstrate the ability to resolve neutron sources separated by a spacing of $1.27 \mathrm{~cm}$, similar to the spacing between fuel pins in commercial pressurized water reactor $17 \times 17$ nuclear fuel assemblies. This report fulfills the deliverables "Technical report on laboratory measurements of source configurations" and "Final Report" for the project "Detection of Fuel Pin Diversion via Fast Neutron Emission Tomography" supported by the Office of Defense Nuclear Nonproliferation Research and Development. The remainder of this report opens with a description of the as-built parallel-slit ring collimator, including the collimator, detectors, data acquisition, and fully assembled instrument. Then, it describes imaging measurements with ${ }^{252} \mathrm{Cf}$ fission neutron sources placed within tubing the diameter of a single fuel pin. Last of all, it reports the results of tomographic reconstructions of measurements of individual and multiple sources. The report closes by detailing expected future work to fully characterize the imager that will be supported by the Nuclear Science and Security Consortium through the University of Tennessee Department of Nuclear Engineering.

\section{THE IMAGER}

While measuring fast neutrons is desirable because neutrons readily escape from a fuel assembly, their ability to penetrate shielding makes fast neutrons difficult to collimate and measure with fine spatial resolution. Measuring the modest neutron source strength from spent fuel among the overwhelming gamma-ray emissions poses additional challenges. The present imager addresses these challenges by using a functional equivalent to a parallel-slit collimator called a "parallel-slit ring collimator." Unlike a true parallel-slit collimator, it can employ many large detector pixels close to the fuel. In this design, the slits of a parallel-slit collimator are rearranged to distribute them around a ring that surrounds the target fuel assembly. Each slit creates the same line of response as it would in its original parallel-slit geometry

but for a different projection angle (rotation of the object). For tomographic measurements, all projection 
angles (spaced around $360^{\circ}$ ) are measured, so an equivalent set of views will be acquired for the rotated slits as for the original parallel slits. This rearrangement enables use of large, moderated detectors that are relatively close to the fuel with widely spaced collimator slits that simultaneously sample the entire volume. Moderated detectors offer the advantage of achieving their neutron efficiency in proportion to the volume of moderator, which is insensitive to gamma rays, while having comparatively little active volume that is sensitive to gamma rays. Use of the parallel-slit ring collimator concept makes it possible to construct an imager with sufficient resolution to identify individual fuel pins and keep the imager sufficiently compact, efficient, and radiation resistant to be practical.

The prototype imager consists of a cylindrical inspection volume, an annular collimator that surrounds the inspection volume, a ring of neutron detectors that surrounds the collimator to detect neutrons that traverse the slits, and a data acquisition that records neutron counts in the detectors. The collimator sits on a gantry that allows the collimator to rotate to acquire projection data at different angles. For this collimator design, the neutron source and detectors can remain stationary, and only the collimator need rotate. As a result, source-detector positioning does not have to be made very precisely provided there is no relative motion during the measurement.

The annular collimator has a set of 72 slits that define lines of response across the imager FOV. The cylindrical inspection volume, which corresponds to the central opening of the annulus, has a diameter of $35.0 \mathrm{~cm}$ and a height of $100 \mathrm{~cm}$. A cross section of the imager design is shown in plan view in Figure 1, where the $100-\mathrm{cm}$ dimension of the inspection volume extends out of the page. In the actual imager, there is also $5 \mathrm{~cm}$ of borated polyethylene shielding on the outer radius of the detectors to reduce the count rate in the detectors from background neutrons or other neutron sources that may be in the same laboratory.
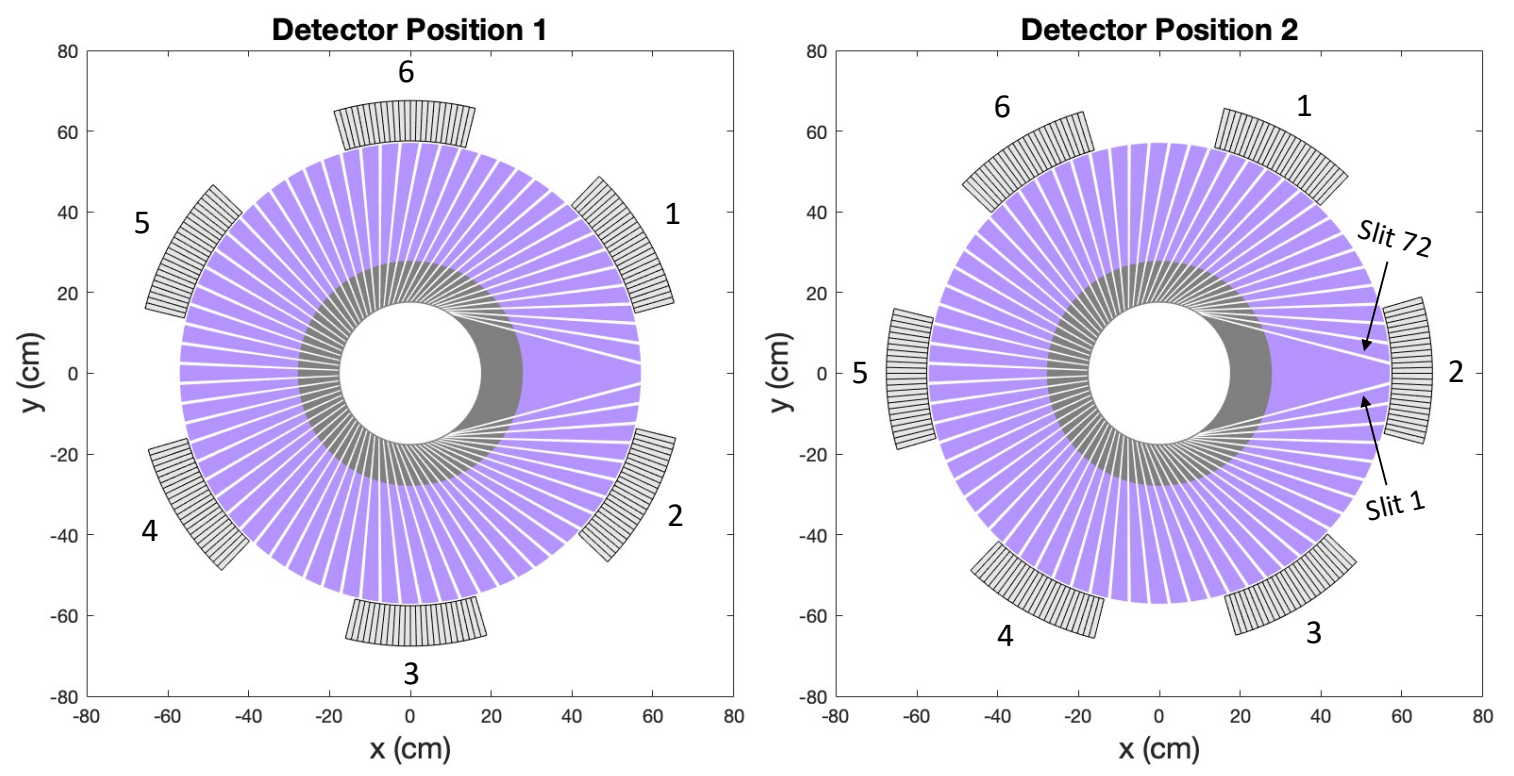

Figure 1. Plan view of the imager design. The collimator annulus is composed of an (grey) inner stainless steel and (purple) outer borated polyethylene ring and has 72 slits in it that define lines of response across the FOV. Neutron counts are recorded in (light grey) 6 detector modules that each have 24 rows of 8 boron straws. The detectors are moved between

Neutron line sources (eventually, spent nuclear fuel) can be loaded in the inspection volume. The collimator modulates neutrons emitted by the fuel, and the modulated pattern of collimated neutrons are detected by the ring of neutron detectors. To perform imaging measurements, the collimator rotates on the 
gantry, and the detectors and line sources remain stationary. Data for each chord across the inspection volume and each angle are recorded, enabling tomographic reconstruction.

The inner diameter of the collimator itself is $35.7 \mathrm{~cm}$. This dimension is intended to accommodate most common light water reactor fuel assemblies. In particular, the inner diameter was chosen such that its inscribed square is $1 \mathrm{~cm}$ greater in side length than an assembly with a width of $24 \mathrm{~cm}$. The innermost 10 $\mathrm{cm}$ of the collimator, shown as dark gray on Figure 1, consists of stainless steel to provide structural strength and gamma shielding. The subsequent $29.22 \mathrm{~cm}$, shown purple, consists of borated polyethylene that is $5 \%$ by weight boron. This portion of the collimator effectively modulates the neutron intensity.

The inner diameter of the detector arc is $115.1 \mathrm{~cm}, 1.0 \mathrm{~cm}$ greater than the outer diameter of the collimator to provide a gap of $0.5 \mathrm{~cm}$ between the two to accommodate the relative motion. The outer diameter of the detector ring is $135.3 \mathrm{~cm}$. Including the $5 \mathrm{~cm}$ of borated polyethylene shielding on the outer diameter of the detectors, the outer diameter of the imager is approximately $146 \mathrm{~cm}$. The outer diameter of the imager was chosen so that it can be readily handled by a facility, so its size was constrained to fit in a cask or cask-like container that nuclear operators typically handle. The outer diameter was therefore chosen to fit within the internal cavity of the $148 \mathrm{~cm}$ internal cavity diameter of the GNS Castor series of transport casks, a cask type commonly used for international fuel shipments.

Each detector module subtends 30 degrees of the ring around the collimator so the detector ring, if fully populated, would consist of 12 modules. Each module consists of 24 rows of 8 boron straw detectors embedded in high-density polyethylene (HDPE) moderator. Tolerance to gamma dose dictated that each row of 8 straws is read separately to yield 24 channels per module and a total of 288 channels for the full ring. This number of detector channels oversamples the number of slits by a factor of four.

For the prototype imager, six detector modules were instrumented and positioned in every other detector slot as shown in Figure 1. Measuring all detector positions thereby requires the detectors to be repositioned.

\subsection{THE COLLIMATOR AND GANTRY}

Mechanically, the collimator is assembled from a set of wedge-shaped septa in a common spool-shaped stainless-steel housing where gaps between the septa create slits that define lines of response on distinct chords across the inspection volume of the imager.

When assembled, the collimator consists of a set of 72 slits that define chords that span the central 34.0 $\mathrm{cm}$ diameter of the inspection volume. The distance between successive chords is $0.479 \mathrm{~cm}$, except for the distance between slits 36 and 37, which is half this distance. This offset ensures that none of the slits interrogates duplicate chords. Note that the design reported in [4,5] used 96 slits, which corresponds to $1 / 3$ the number of neutron detector readout channels needed to instrument a full detector ring. The cost of the collimator scaled with the number of septa; when building the collimator, some cost reduction was needed to fit within the project budget. As a result, the number of slits was reduced from 96 to 72 , which corresponds to $1 / 4$ the number of neutron detector readout channels needed to instrument a full ring.

The slits were designed to have a width of $3 \mathrm{~mm}$ at the inner diameter of the collimator and a width of 8 $\mathrm{mm}$ at the outer diameter of the collimator. Because the slits follow different chords across the annulus, they have different lengths and opening angles. Likewise, the septa making up the collimator have unique lengths and opening angles. Each septum is made up of a stainless-steel portion nearest the collimator inner diameter and a borated polyethylene portion that spans the rest of the length to the collimator outer diameter. 
Deflection due to warping of the septa after machining scales like the cube of the length, so to maintain tolerance for the slit width, the steel portion of the septa were constructed from two pieces that are screwed together at the center. A photograph of nickel-plated carbon steel half pieces of the septa is shown in Figure 2 (a). Each half septum was machined to leave raised alignment stops proud of the surface that, when stacked with other septa, ensured the slit gap was maintained. The steel septa were also machined with a lip and threaded holes to affix the borated polyethylene portion of the septum. Example assembled composite septa are shown in Figure 2 (b).
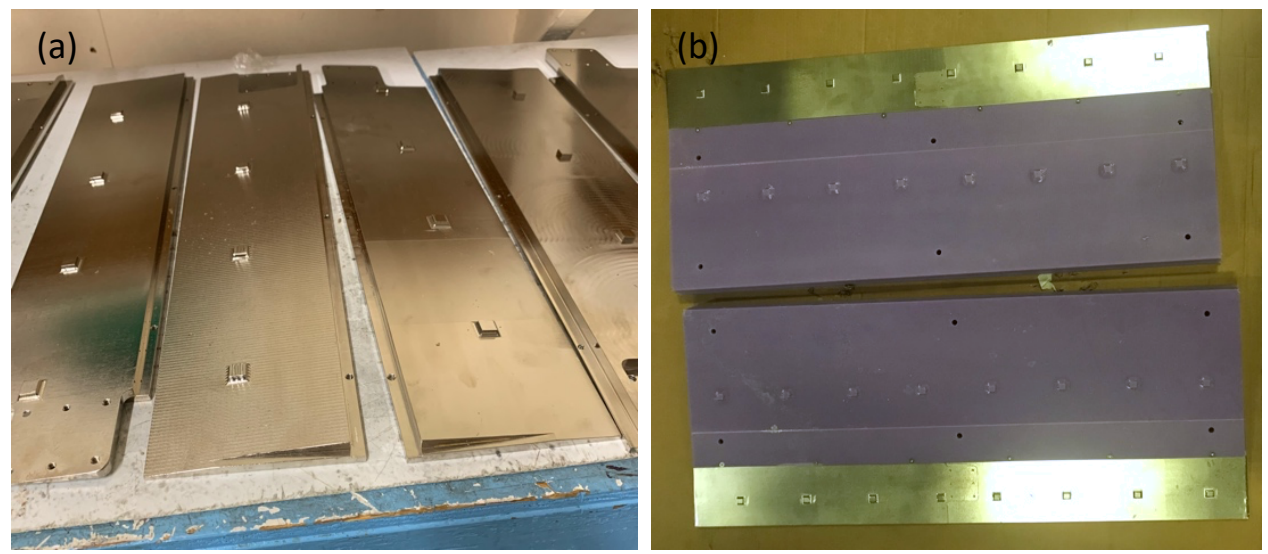

Figure 2. The collimator septa during assembly showing (a) the nickel-plated steel portions and (b) the assembled composite steel and borated polyethylene septa.

The composite septa were then installed in the spool piece, shown in the photograph in Figure 3 (a). Prior to installation of the septa 10 -cm-thick annular pieces of steel and borated polyethylene with guide slits to align the septa were installed in the top and bottom of the spool piece. These pieces ensured alignment of the septa and provided shielding of the collimator slits from above and below. A photograph with the guide pieces installed is shown in Figure 3 (b). A photography of the collimator with nearly all the septa installed is shown in Figure 3 (c).
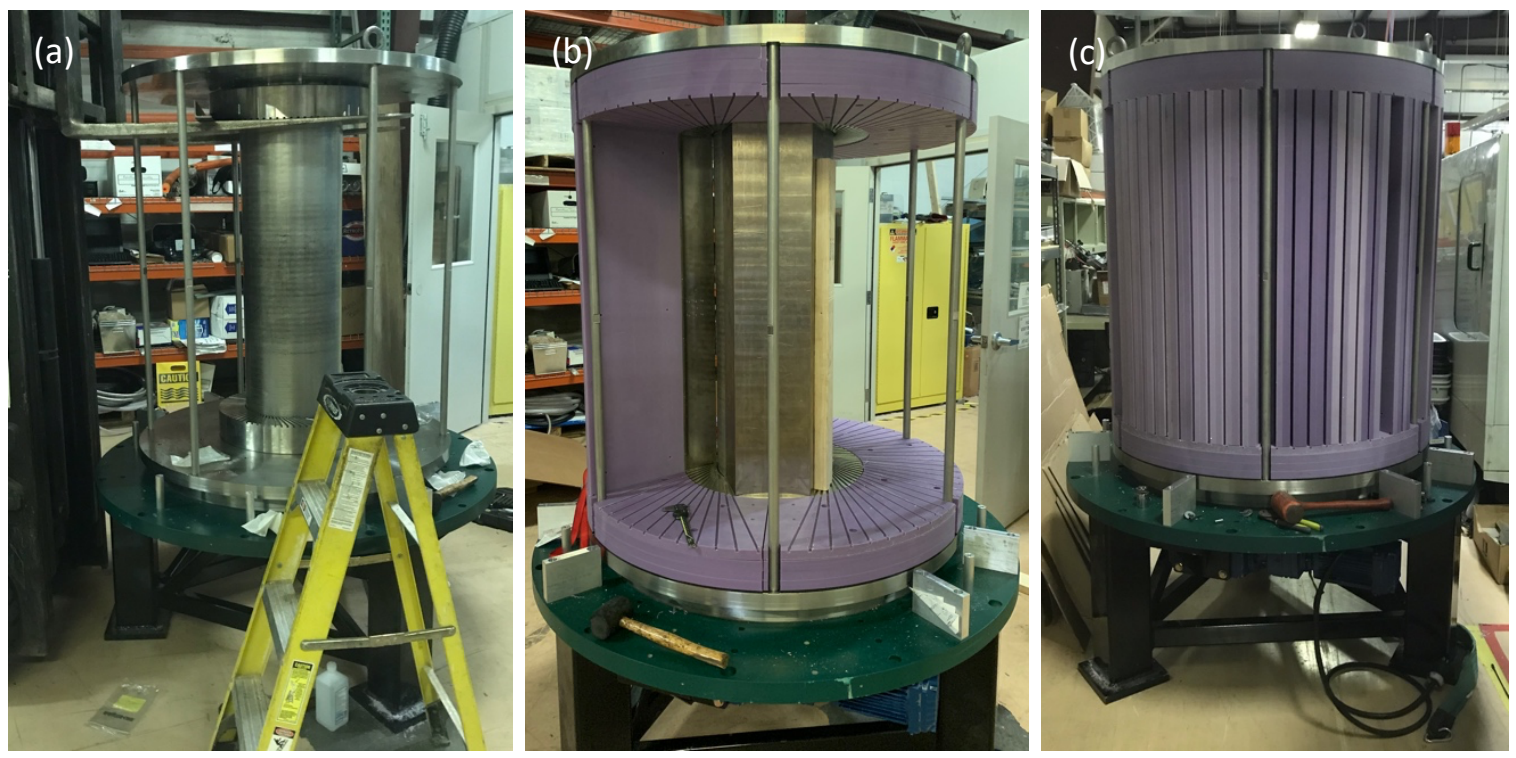

Figure 3. Assembly of the collimator. 
The mechanical design of the gantry assembly enabled its utilization while attached to a floor-mounted support frame or while suspended from rigging. The detector modules are mounted to the primary baseplate of the detector assembly. The collimator assembly is mounted coaxially relative to the stationary detector array on a large diameter high-capacity slewing gear bearing, Kaydon Model \#HS6$37 \mathrm{~N} 1 \mathrm{Z}$, so that it is rotatable. The gear bearing has a pitch diameter of 33.50 inches and is driven by a matching pinion with a pitch diameter of 4.5 inches, resulting in a gear ratio of approximately $7.44: 1$. Rotation of the collimator is driven and controlled by a helical-bevel gearmotor and motion control inverter drive, SEW Eurodrive Model KA67R37** and MC07B0015-2B1**, which has a maximum output rotation of $14 \mathrm{rpm}$. The drive motor and gear ratio provide the capability of varying the rotation speed of the collimator from 0 to approximately $2 \mathrm{rpm}$.

\subsection{THE DETECTORS}

The prototype imager employs 6 identical detector modules, each containing 192 "boron straw detectors" organized in 24 rows of eight straws distributed radially. Each module comprises one twelfth of a ring; the font face of the detector has a radius of curvature of $57.63 \mathrm{~cm}$ and the back face has a radius of curvature of $67.65 \mathrm{~cm}$. The height of the HDPE moderator in the module is $104.5 \mathrm{~cm}$. Each straw in a module is an approximately $4 \mathrm{~mm}$ diameter gas-filled aluminum tube that contains a ${ }^{10} \mathrm{~B}_{4} \mathrm{C}$-coated copper straw at the outer diameter and an anode wire in the center. Neutrons are detected via the neutron capture reaction ${ }^{10} \mathrm{~B}+n \rightarrow{ }^{7} \mathrm{Li}+\alpha$ reaction when either the $0.84 \mathrm{MeV}{ }^{7} \mathrm{Li}$ or $1.47 \mathrm{MeV} \alpha$ escapes the ${ }^{10} \mathrm{~B}_{4} \mathrm{C}$ coating and creates sufficient ionization in the fill gas of the straw to exceed the detector threshold. The straws are resilient to high gamma-ray fields because (1) most of the detector consists of inert moderator, (2) the small gas volume per straw and low gas pressure minimize the amount of material where gamma rays (and scattered electrons) can interact, and (3) the small radius of the detector means that the signals are inherently fast (tens of nanoseconds rather than microseconds) to minimize the amount of gamma-ray pileup. The ${ }^{10} \mathrm{~B}$ straws are manufactured by Proportional Technologies Inc. Although the detectors detect neutrons at thermal energies, by incorporating boron in the collimator and placing a $0.053-\mathrm{cm}$-thick cadmium thermal-neutron filter between the collimator and detector, the imager responds primarily to fast neutrons. As instrumented, the boron straw detectors can tolerate gamma fields up to approximately $500 \mathrm{R} / \mathrm{h}$.

In the detector modules, each row of eight straws is read out by a single, low power electronic front-end readout board. The readout electronics is implemented by a fast current preamplifier as front-end element, followed by an amplification stage, a passive 6-pole low-pass filter with a $56 \mathrm{MHz}$ bandwidth, and a driver. A simple Robinson baseline restorer completes the analog readout chain. The resulting analog signal triggers a comparator with a threshold common to the entire readout module. Such signal indicates that any of the eight straw detectors that feed the readout channel has seen an event. The comparator's output uses low voltage differential signaling (LVDS) and is sent to a processing board. The processing board is a single printed circuit board that spans the entire top surface of the detector module. It provides several common functions. It receives, buffers, and re-broadcast the digital LVDS signals from the frontend boards, it provides the comparator threshold distribution to the front-end boards, and it distributes the detector bias voltage to the straw detectors. The processing board also accepts a $\pm 6.5 \mathrm{~V}$ direct current (DC) bias and provides the low voltage bias necessary for the logic as well as the front-end board to operate ( $\pm 5 \mathrm{~V}$ and $3.3 \mathrm{~V}$ to the front-end board and $3.3 \mathrm{~V}$ digital for the on-board logic). The output signals are provided for further processing to the back end and data acquisition (DAQ) electronics via Cat6e shielded cables. This solution ensures wide bandwidth pipe to the back end while using inexpensive, reliable, commercial parts. Of note is the fact that the pin assignment for the RJ-45 connectors respects the EIA568-B standard, so that common Cat6e cables may be used for signal transmission, allowing signal broadcast over long cables. This sets the channel numbering for each module. Analog channels are numbered from 1 to 24 starting from the left side when looking at the detector module from the fuel pin perspective. Each RJ-45 connector carries signals from four front-end 
boards (LVDS is differential signaling, thus, each analog channel outputs a pair of comparator signals) so that six such connectors serve the entire detector module. By using the EIA568-B encoding, pin 1 of the RJ-45 connector is channel 1, pin 2 is the complement of the same channel, pin 3 is the complement of channel 2, pin 6 is channel 2, pin 4 and 5 are respectively channel 3 and the complement of channel 3 , and, finally, pin 7 and 8 are the complement of channel 4 and channel 4 . As long as the same convention is applied at the receiving (back) end, no further considerations regarding channel numbering is needed. Special attention was given to providing the lowest impact on power consumption via proper component choice. The resulting current required by a single detector module is $1.38 \mathrm{~A}$ from the positive bias and about $0.46 \mathrm{~A}$ from the negative bias voltage. Photographs of a detector module during assembly are shown in Figure 5.
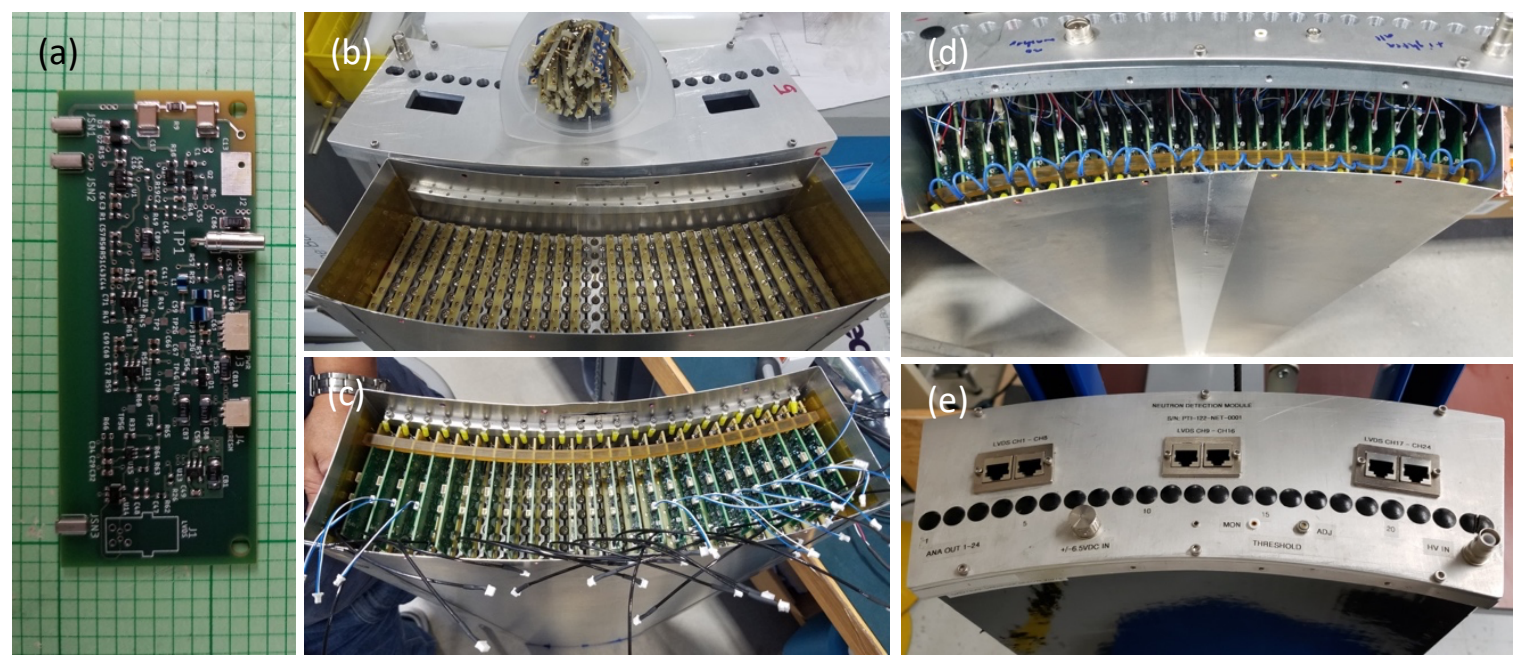

Figure 4. A detector module during assembly, including (a) a preamplifier board for a row of straws and a module with (b) the straw ends with grounding and connector sockets visible, (c) the 24 preamplifiers installed, (d) the high voltages and discriminator outputs connected, and (e) the module lid in place.

The set of 6 detectors was mounted in a ring with spacers so that every other detector position was populated. Photographs of the six modules mounted on the mounting ring are shown in Figure 5 (a), and with the addition of borated polyethylene shielding and detector cabling in Figure 5 (b).
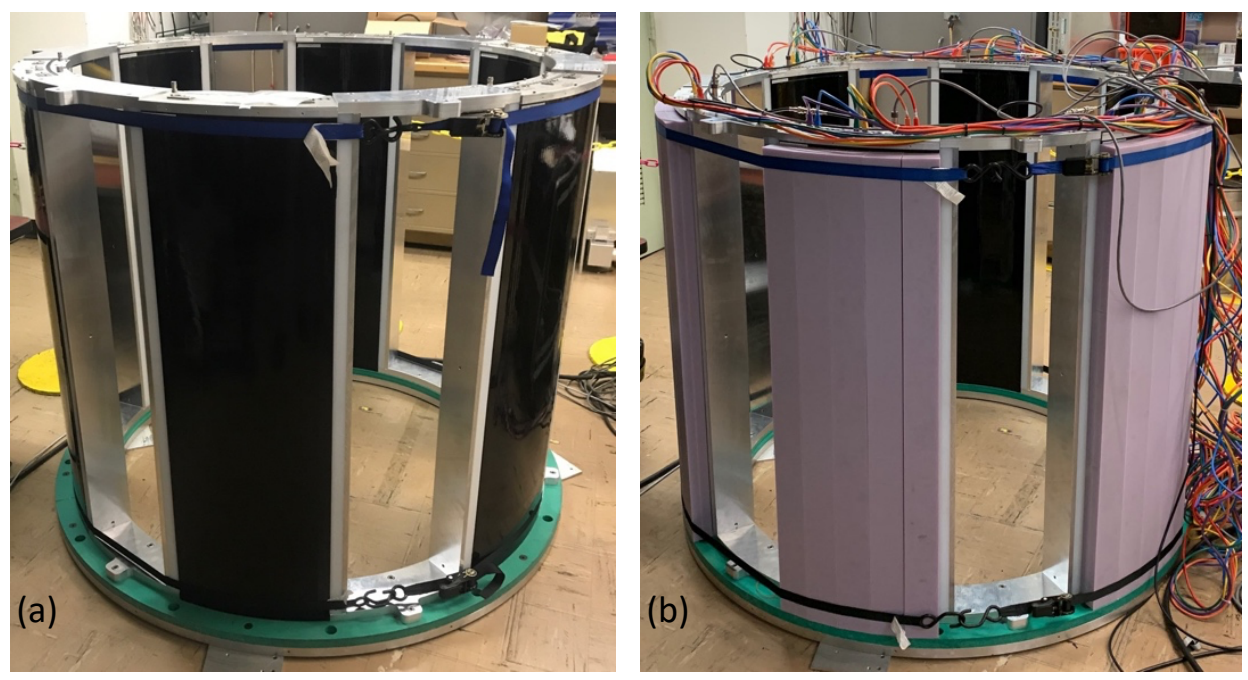

Figure 5. The set of 6 detectors (a) mounted with spacers to form a complete ring, and (b) with the addition of shielding, power, and logic signal cables during detector testing. 


\subsection{THE DATA ACQUISITION}

The DAQ is based on an off-the-shelf CAEN V2495 Programmable Logic Unit with three A395A digital input piggyback boards and the FW2495SC FPGA firmware to enable use as a multievent latching scaler housing 160 independent counting channels. This unit is sufficient to read all 144 detector channels in a single, 1-unit wide VME 6U module. In addition, there are 16 spare channels to enable input of a clock pulser and optical switches to encode the position of the collimator in a common data stream. The V2495 is read over the VME bus via a Struck SIS3153 bridge via custom software procured from Cadre5. This DAQ software also provides for control of two CAEN V6533P six-channel, positive polarity, $4 \mathrm{kV}, 3 \mathrm{~mA}$ high voltage (HV) power supplies. A photograph showing the four slot, 2U VME chassis with the V2495 logic module, two V6533P HV modules, and SIS3153 VME bridge is shown in Figure 6.

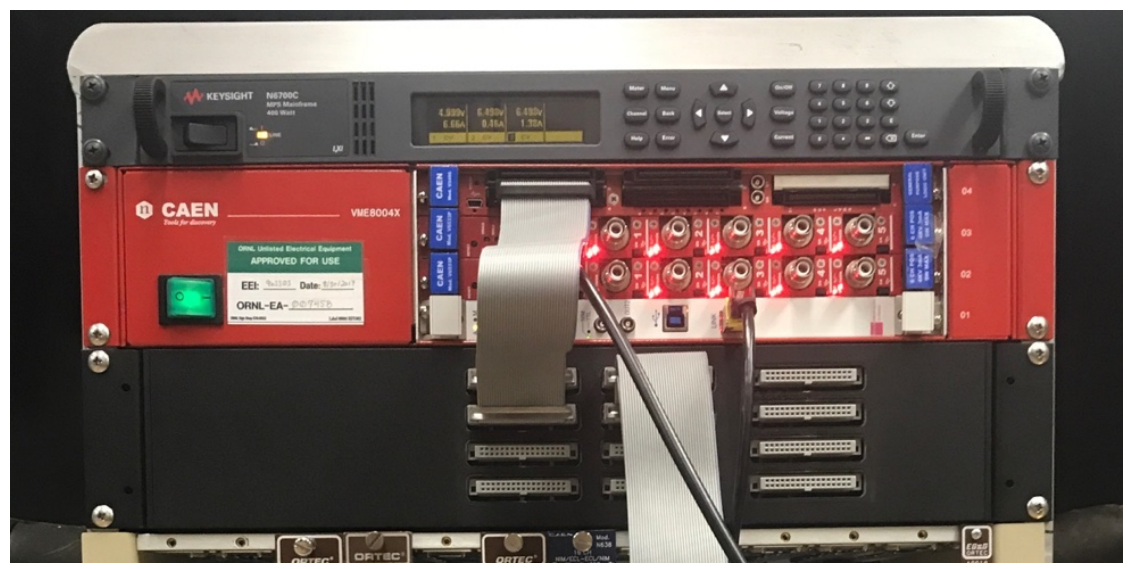

Figure 6. The data acquisition system.

The V2495 modules accept LVDS input signals on proprietary CAEN A967 flat ribbon cables. These cables are not ideal for transmission over long distances (meters), but our choice of Cat6e cables allows the DAQ to be approximately $10 \mathrm{~m}$ away from the detectors. As a result, a custom interface module was designed to convert Cat6e signaling into flat ribbon cable near the CAEN modules. This interface is located inside a 19" rackmount enclosure. It has 48 RJ-45 inputs (thus, it can serve up to 192 channels of readout) distributed over four PC boards, internal to the module. The interface circuits are straightforward: the LVDS signals coming from the detector module are buffered and driven into a CAEN-proprietary flat ribbon cable that, at the far end, matches the 64-pin connectors present on the CAEN module, and on the near end has two standard 34 position (two positions are unused on each connector), dual row rectangular connector. This means that each flat-ribbon cable carries a total of 32 signals. There are 12 rectangular connectors in the interface module for a total of 192 signals to match the total number of inputs. Two interface modules will easily serve 12 detector modules with many spare channels. The timing section of the DAQ requires by design a constant clock to be available, presently set at $32.768 \mathrm{kHz}$, therefore, one channel every third header can be optionally used to transmit the clock signal generated on the interface board. Generally, these are the leftmost headers in the module front panel. The selection of whether to send a detector signal or a clock signal is done via jumpers.

Due to the mechanical characteristics of the CAEN flat-ribbon cable, a straight-through mapping of the channel numbers was not possible (see cabling diagram in Figure 7). 


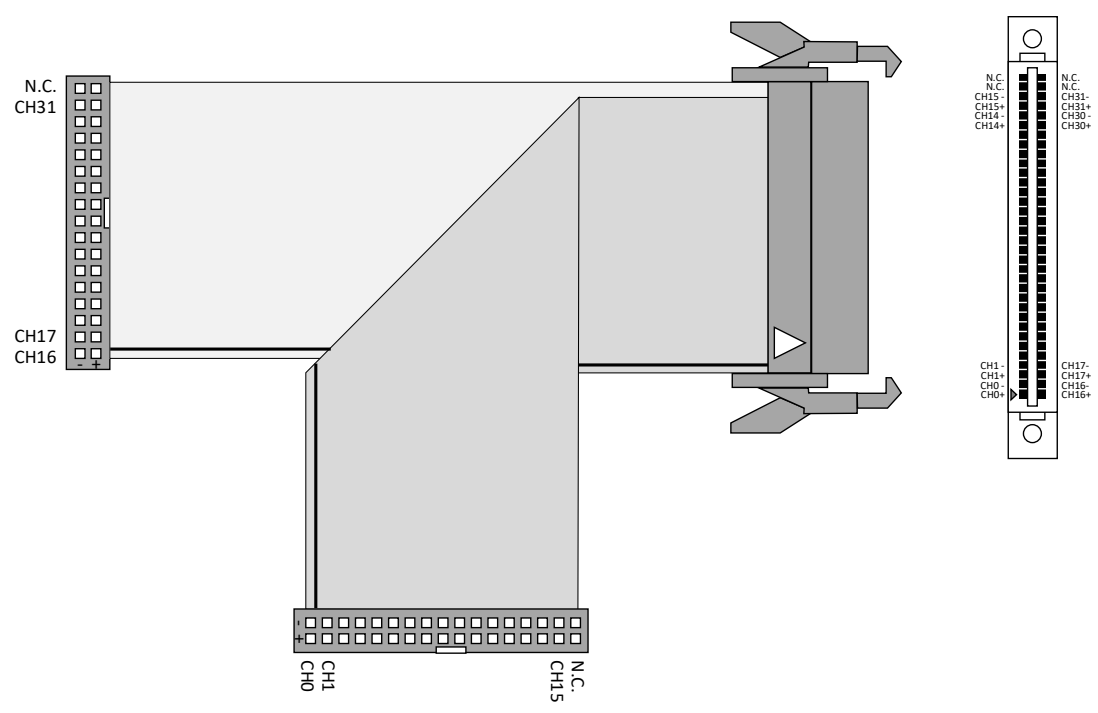

Figure 7. CAEN A967 flat ribbon cable, “68-pin P50 connector to two $2.54 \mathrm{~mm} 34$ pin male connectors" (after https://www.caen.it/products/a967/)

The best way to accommodate easy connection of this cable is to plug the 34 pins rectangular connectors starting from the right side of the module. The first connector plugs to the lower rightmost mating connector on the panel. The second plugs under this connector. The next cable plugs in the center row according to the same modality and so on. These connectors are assigned letters A-F to differentiate to which connector a reference is being made. The lower rightmost mating connector is labeled $\mathrm{A}$, while the lower center connector is $\mathrm{B}$ and the leftmost lower connector is labeled $\mathrm{C}$. The upper rightmost connector is labeled $\mathrm{D}$, the center is labeled $\mathrm{E}$, and the upper leftmost is labeled $\mathrm{F}$. Consequently, the Cat6e cable on the opposite side of the module must be mapped accordingly.

Due to the design of the CAEN DAQ, the connector labeled C is designated as a set of 32 output channels. For this reason, no RJ-45 cables are connected to that connector. The back of the CAEN module has 48 RJ-45 inputs arranged in grid of 4 rows by 12 columns. Within the grid, each of the 6 connectors has 8 inputs arranged in a grid of 2 rows by 4 columns, as shown in Figure 8 . To read signals out from the detector modules, the RJ-45 inputs for the first detector are connected to Connector A. Beginning with the $2 \times 4$ grid starting in the lower rightmost corner of the module, the first RJ-45 cable is plugged into the lower left input of the 8 inputs in this group. The connections continue in this row of 4 from right to left before jumping to the upper row of 4 inputs and continuing left to right. The connections then go to the lower center $2 \times 4$ grid of inputs, corresponding to Connector B, and continue in the same pattern of connecting to the lower row, going from left to right, then the upper row left to right. Each RJ-45 input from each detector module is mapped in this pattern to the alphabetically advancing connector, skipping connector $\mathrm{C}$.

The power requirement of the interface module is approximately $8 \mathrm{~A}$ from a $3.3 \mathrm{~V}$ supply. To power the entire system, a Keysight N6700C power system was chosen. This system is a 19" modular rack that can host a combination of power supplies. A single rack (N6700C) with two N6742B (8V, 12.5A for the detector module) and one N6731B (5V, 10A for the interface box) powers six detector modules and the interface module. 


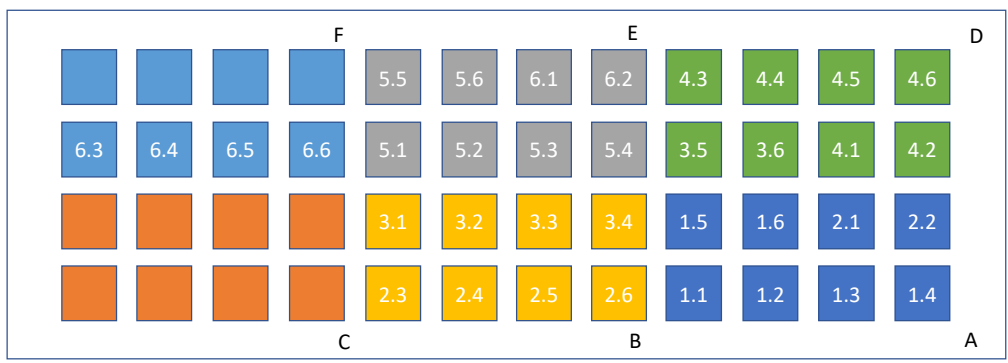

Figure 8. Wiring diagram for the translator.

\subsection{THE ASSEMBLED IMAGER}

The imager was assembled from the rotatable collimator on its floor-mounted support stand, the motor and controller, the detectors, and the data acquisition. The diameter of the imager mounted on the floor stand with detectors and shielding installed is approximately 1.46 meters (57.50 inches) and the height is approximately 1.47 meters (58.0 inches). A photograph of the operational imager is shown in Figure 9.

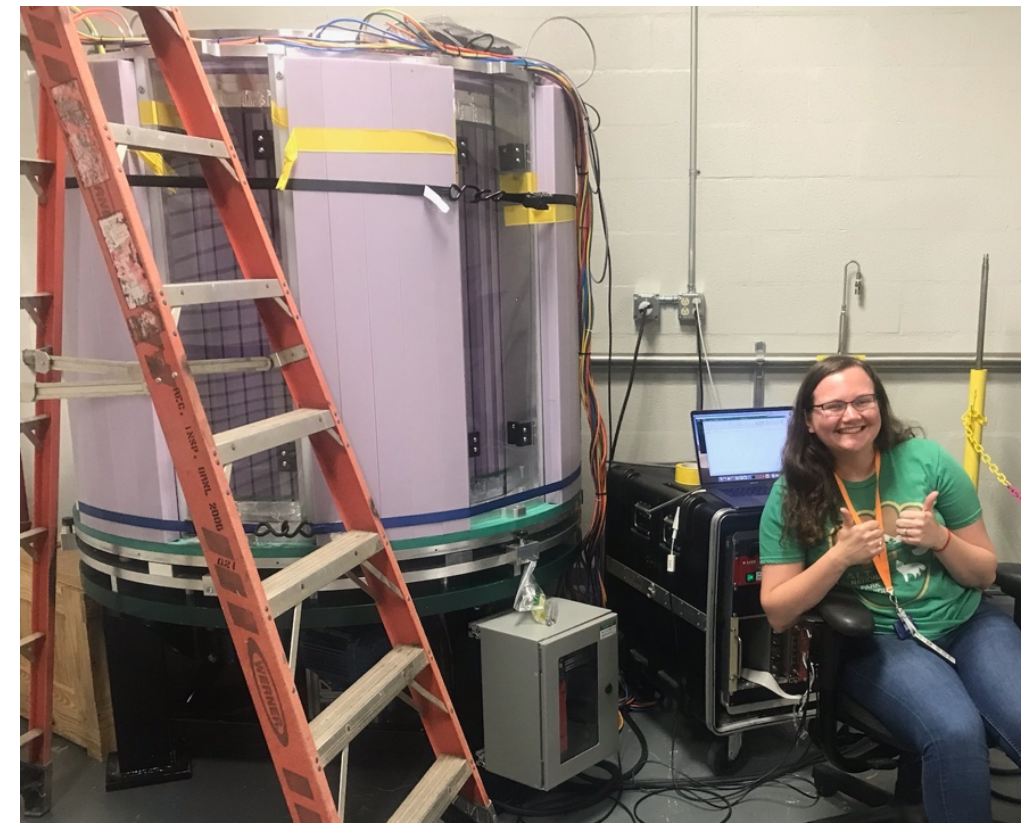

Figure 9. The assembled imager.

The detectors were mounted on an annular platform which is free to spin on ball transfers to allow movement of the detectors between measurement positions. The detector platform can be pinned at increments of 30 degrees rotation to ensure repeatability in detector placement. The spacers between the detectors were fastened to the annular platform and the detectors slid into the openings between spacers. The back sides of the detectors were shielded by $5 \mathrm{~cm}$ of borated polyethylene (purple in Figure 9), and the detectors and shielding were held in place against the spacers by ratchet straps similarly to the staves of a barrel. Collimator septa can be seen through the central openings in the spacers. The spacer openings were covered by acrylic windows to shield fingers or clothing from catching in the rotating collimator.

The motor power supply and controller were installed in a National Electrical Manufacturers Association rated electrical enclosure and mounted to a leg of the imager support stand. DAQ components and detector power supplies were mounted in a 10U 19-inch rack. Data were recorded by a Macbook Pro laptop computer connected via Ethernet to the Struck SIS 3153 VME bridge. 


\section{MEASUREMENTS}

Initial measurements were performed to demonstrate working operation of the imager and to assess whether the imager can resolve fission neutron sources spaced at distances comparable to the spacing of fuel rods in $17 \times 17$ commercial pressurized water reactor nuclear fuel assemblies. As a result, a fixture was designed and built to mimic aspects of such an assembly. The fixture consisted of a table that supports two aluminum plates, each with a $17 \times 17$ array of $0.9652 \mathrm{~cm}(0.380$ inch $)$ holes with the centers spaced at $1.27 \mathrm{~cm}(0.500 \mathrm{inch})$ intervals. Four of the holes in each plate were used to accommodate rods that hold the plates at a fixed separation of $94 \mathrm{~cm}$. The bottom plate in the fixture was located $30 \mathrm{~cm}$ below the centerline of the imager's inspection volume.

A set of $121.9 \mathrm{~cm}$ (48 inches) long, $0.9525 \mathrm{~cm}$ (0.375 inch) diameter, stainless-steel rods was also procured. These rods can populate the fixture to mimic the attenuation of fuel rods in a fuel assembly. A drawing of the fixture, populated with steel rods and positioned in the imager, is shown in Figure 10 (a). A cutaway along the centerline of the imager is shown in Figure 10 (b). A rendering of the fixture only is shown in Figure 10 (c).

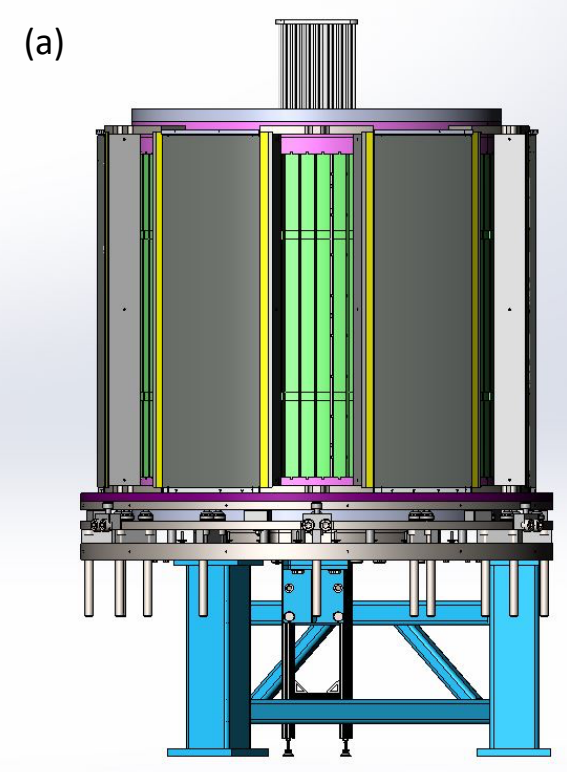

(b)

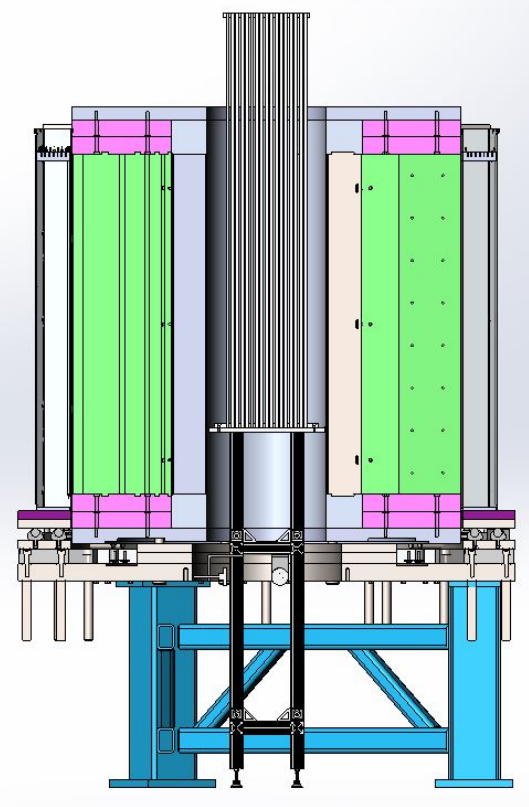

(c)

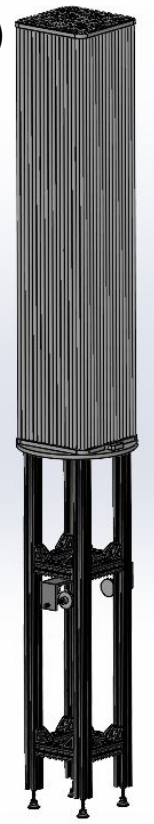

Figure 10. Fixture that mimics a $17 \times 17$ PWR nuclear fuel assembly

A neutron source rod was constructed from $0.9525-\mathrm{cm}(0.375 \mathrm{inch})$ diameter thin-walled aluminum tubing and two $0.6-\mathrm{cm}$-diameter ${ }^{252} \mathrm{Cf}$ fission neutron sources. An elastomer stop was inserted into the tubing $25 \mathrm{~cm}$ from the bottom such that, when the sources were placed in the tubing, the location of the sources resting on the stop was $3 \mathrm{~cm}$ below the centerline of the imager's inspection volume. The two ${ }^{252} \mathrm{Cf}$ sources, Cf-252-5442 and Cf-252-5443, had emission rates estimated to be 61090 and 6690 neutrons per second for the time of the measurements, for a total of 67780 neutrons per second.

The source rod could be moved from position to position in the fixture to perform tomographic measurement of a particular neutron source position. Multiple-source configurations were measured by measuring the individual source positions with a single source and then summing the data from the multiple measurements. In practice, simultaneous measurements with several sources will be possible, but simultaneous measurements with hundreds of sources would be both cost and dose prohibitive. For the 
present measurements, five positions were chosen with a range of separations to enable evaluation of the resolution of the imager. Using a numbering scheme where $(0,0)$ is the coordinate of the center slot in the fixture, measurements were performed for slots $(0,0),(0,4),(2,0),(1,3)$, and $(2,1)$. This set of measurements allowed investigation of pairs of sources that were separated by distances of 1

(immediately neighboring), $\sqrt{2}, 2, \sqrt{5}$, and 4 slots. A photograph of the top of the imager with the fixture visible in the inspection volume is shown on the left of Figure 11. A close-up of the top of the source fixture with the source rod positioned in slot $(2,0)$ is shown on the right of Figure 11.
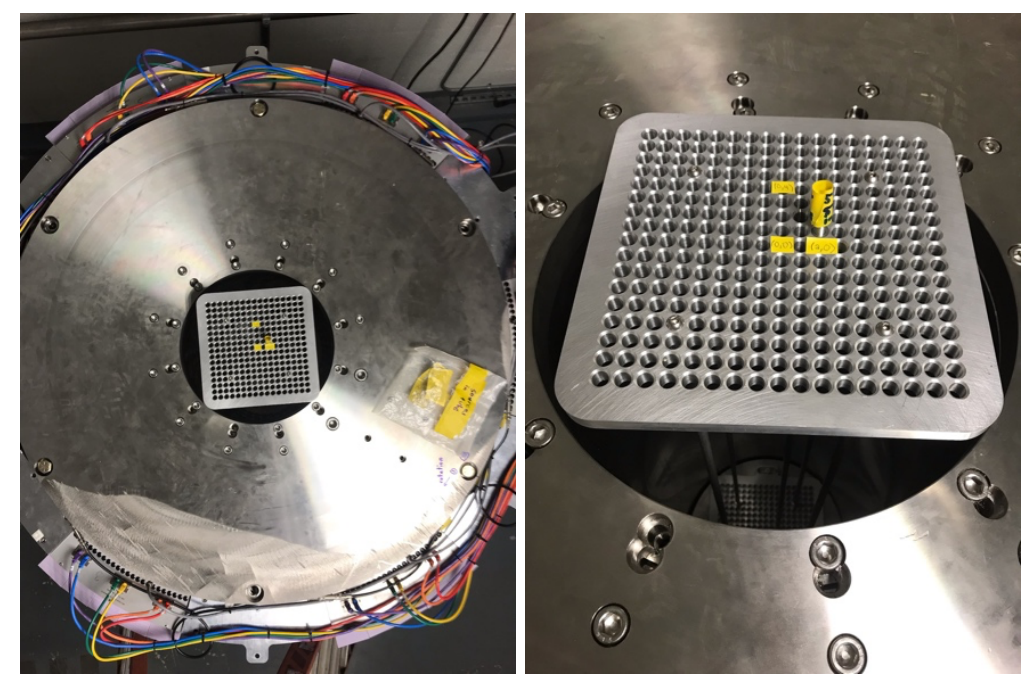

Figure 11. Fixture that mimics $17 \times 17$ PWR nuclear fuel assembly.

Details of the measurements, including date of measurement, measurement duration, emitted neutrons per projection, and detected neutrons per projection are summarized in Table 1.

Table 1: Summary of initial measurements performed.

\begin{tabular}{c|c|c|c|c}
$\begin{array}{c}\text { Source } \\
\text { slot }\end{array}$ & $\begin{array}{c}\text { Date of } \\
\text { Measurement }\end{array}$ & $\begin{array}{c}\text { Measurement } \\
\text { time }(\mathrm{s})\end{array}$ & $\begin{array}{c}\text { Emitted neutrons } \\
\text { per projection }\end{array}$ & $\begin{array}{c}\text { Detected neutrons } \\
\text { per projection }\end{array}$ \\
\hline$(0,0)$ & $6 / 28 / 21$ & 4520 & $4.26 \times 10^{6}$ & 7571 \\
$(0,4)$ & $7 / 1 / 21$ & 4641 & $4.37 \times 10^{6}$ & 7460 \\
$(2,0)$ & $7 / 1 / 21$ & 4518 & $4.25 \times 10^{6}$ & 7454 \\
$(1,3)$ & $7 / 12 / 21$ & 4593 & $4.32 \times 10^{6}$ & 7450 \\
$(2,1)$ & $7 / 2 / 21$ & 4606 & $4.34 \times 10^{6}$ & 7485
\end{tabular}

An example projection measurement is shown in Figure 12. This projection is for the collimator at its starting angle and the neutron source located at position $(0,0)$. The duration of acquisition for this projection was $62.8 \mathrm{~s}$. A total of 7315 neutrons were detected during this time which corresponds to $1.78 \times 10^{-3}$ of the emitted source neutrons. Of the detected neutrons, approximately $917 \pm 40$ are in the peak associated with the line of response rather than penetration of the collimator which corresponds to about $2.16 \times 10^{-4}$ of the emitted neutrons. The peak position for a source centered in the inspection volume corresponds predominantly to the detectors behind slit 37 . 


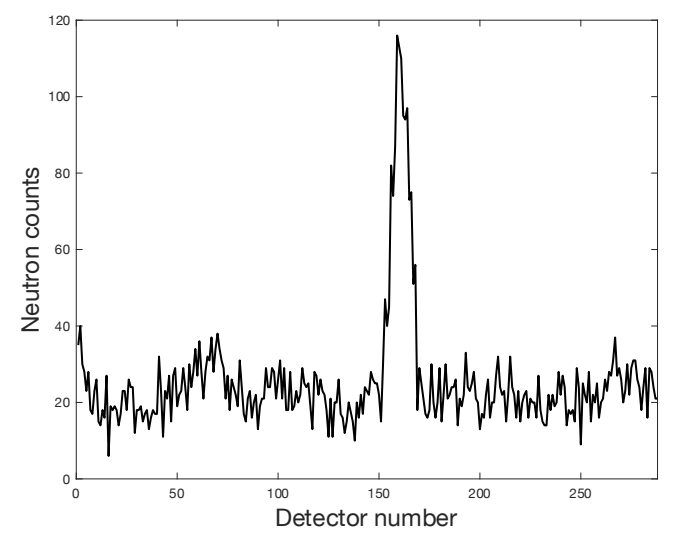

Figure 12. Example projection for neutron source at position $(0,0)$ and collimator at initial rotation angle.

To perform tomographic measurements, the collimator was rotated at a constant angular speed while data were recorded. Then, the neutron count data was binned by collimator position. For the present report, the data were binned in a number of projection angles equaling the number of collimator slits. The neutron counts were collected as a two-dimensional histogram with detector number on one axis and projection number on the other axis; tomographic data presented in this way is normally referred to as a "sinogram" because in parallel-beam computed tomography, each voxel of the object corresponds to a sine wave on the sinogram with the amplitude and phase determined by the distance from the center of rotation and the position on a circle of that radius. In the present context, the sinogram is less straightforward to interpret because each slit measures a different projection angle. Example measured sinograms are given in Figure 13 for the neutron source located at positions $(0,0),(0,4)$, and $(2,0)$.
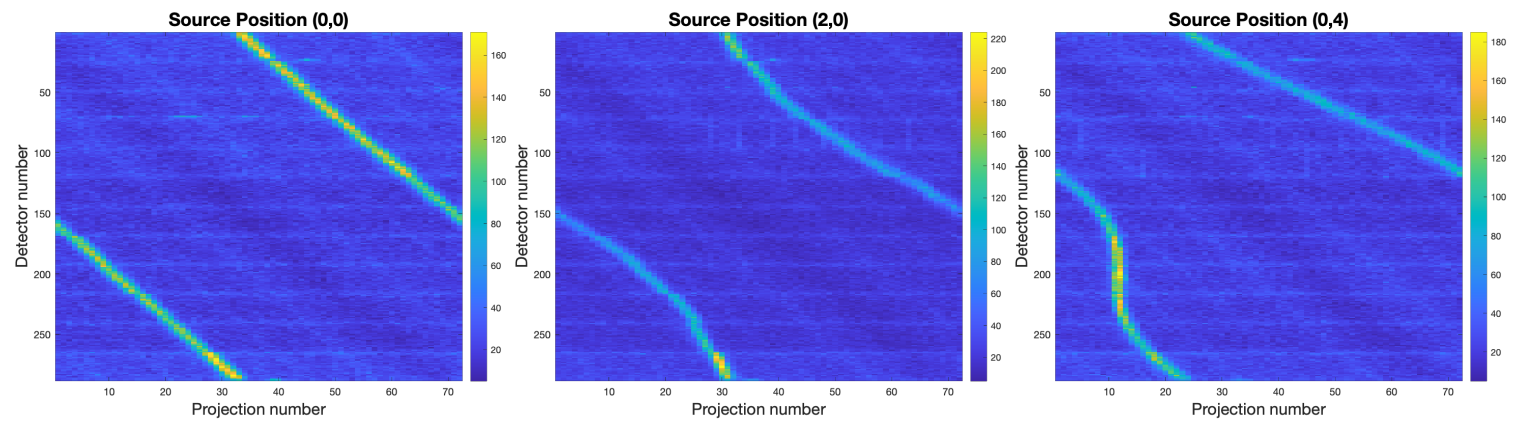

Figure 13. Example sinograms for source positions $(0,0),(2,0)$, and $(0,4)$.

\section{IMAGE RECONSTRUCTION}

Supposing an image $\mathbf{x}$ whose pixel values $x$ correspond to the emitted neutron intensity for a voxel in space, measured projection data (i.e., a sinogram) $\mathbf{y}$ with values $y_{0}$ that correspond to detected counts for each detector and rotation of the collimator, and the imager response or system matrix $\boldsymbol{A}=[a]$ such that $\boldsymbol{A} \boldsymbol{x}=\boldsymbol{y}$, or equivalently, $y=\sum a x$, then image reconstruction is the process of solving for $\boldsymbol{x}$ given $\boldsymbol{y}$ and $\boldsymbol{A}$. Generally, $\boldsymbol{A}$ is ill-conditioned, making the explicit solution a poor choice because it will compound errors in $\boldsymbol{y}$. There are many methods to solve the problem iteratively, and all require the ability to "forward project" a guessed image to produce a trial sinogram. In the present work, images were computed iteratively using the maximum likelihood expectation maximization (MLEM) algorithm for 1000 iterations. The MLEM algorithm is derived from formally maximizing the log likelihoods, assuming 
the values $\boldsymbol{y}$ to be Poisson distributed about the solution $\boldsymbol{A x}$. This maximization results in an update equation for the $\mathrm{k}+1^{\text {st }}$ image values $x_{j}^{(k+1)}$ that is multiplicative.

$$
x_{j}^{(k+1)}=x_{j}^{(k)} \frac{1}{\sum_{i} a_{i j}} \sum_{i} a_{i j}\left(\frac{y_{i}}{\sum_{h} a_{i h} x_{h}^{(k)}}\right)
$$

In words, the $k^{\text {th }}$ trial image is forward projected to produce a trial sinogram. Then, the ratio of the measured-to-calculated sinograms is back projected to update the image by the weighted average of all the sinogram points connected to a particular image point. The derivation of the update equation is valid if the $y_{i}$ are Poisson distributed. The response $\boldsymbol{A}$ for the imager is calculated as described in [5]. At present, detailed investigation of convergence criteria and speed of convergence was not performed. These investigations await tuning the response $\boldsymbol{A}$ so that the forward projection of the true source configuration $\boldsymbol{A} \boldsymbol{x}$ accurately reflects the measured sinogram.

\subsection{RECONSTRUCTION OF MEASUREMENTS OF INDIVIDUAL SOURCES}

In this section, reconstructions will be shown for source positions $(0,0),(0,4)$, and $(2,0)$.

\subsubsection{Position $(0,0)$}

The (left) measured and (right) reconstructed sinograms for the source positioned at $(0,0)$ are shown in Figure 13. The data were reconstructed using the MLEM algorithm for 1,000 iterations. This source is roughly centered in the inspection volume, so the trajectory of regions of high count rates across the sinogram is a straight line. Also note the horizontal lines apparent in the data; these locations correspond to detector edges. For the present analysis, the relative efficiency of the detector in the calculated response was increased near its edges to reproduce this feature. This increased efficiency was attributed to the 2.54 $\mathrm{cm}$ thickness of HDPE adjacent to the detector that could scatter in additional neutrons. The magnitude of count rates associated with the detector edges suggest that these neutrons are associated with source. However, the lack of increased rate when the detector edge views the source (compare modulation of neutron counts in the measured and reconstructed sinograms) suggests that the neutrons get to the detector by a path that does not include the slit directly viewing the source. A more appropriate way to calculate the additional edge response (such as a given fraction of the sum of counts in all detectors) will be investigated in the future.
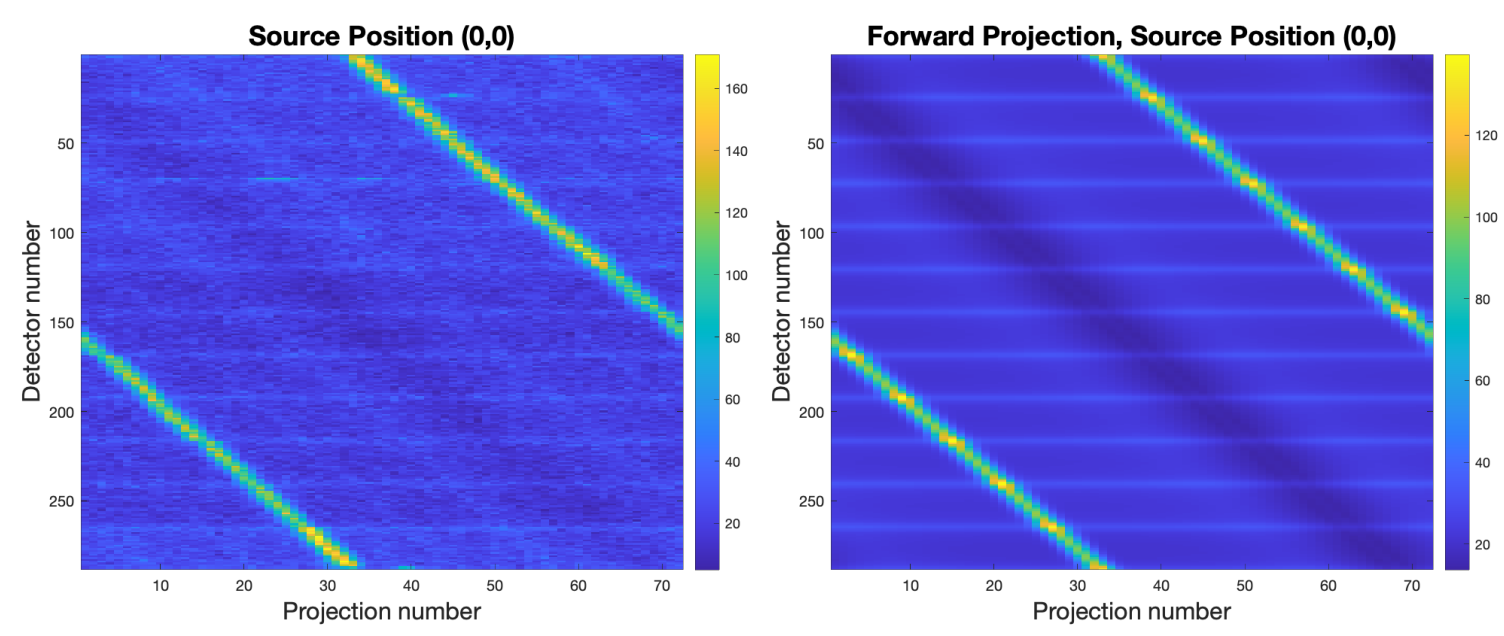

Figure 14. The (left) measured and (right) reconstructed sinogram for source position $(0,0)$ 
Visual comparison of the measured and reconstructed sinograms in Figure 14 enables assessment to determine whether sinogram features are reproduced by the reconstruction, but quantitative assessment is more easily accomplished in one-dimensional plots. As a result, the (black) measured and (red) reconstructed detector counts for projection numbers 10, 20, 30, 40, 50, and 60 are shown in Figure 15. Note that the peak counts are well reproduced in projections 10 and 20 but appear between $20 \%$ and $40 \%$ off in projections 30,40 , and 50. A top priority of future characterization will be to perform efficiency calibrations of the detectors and slits to calibrate any such differences.
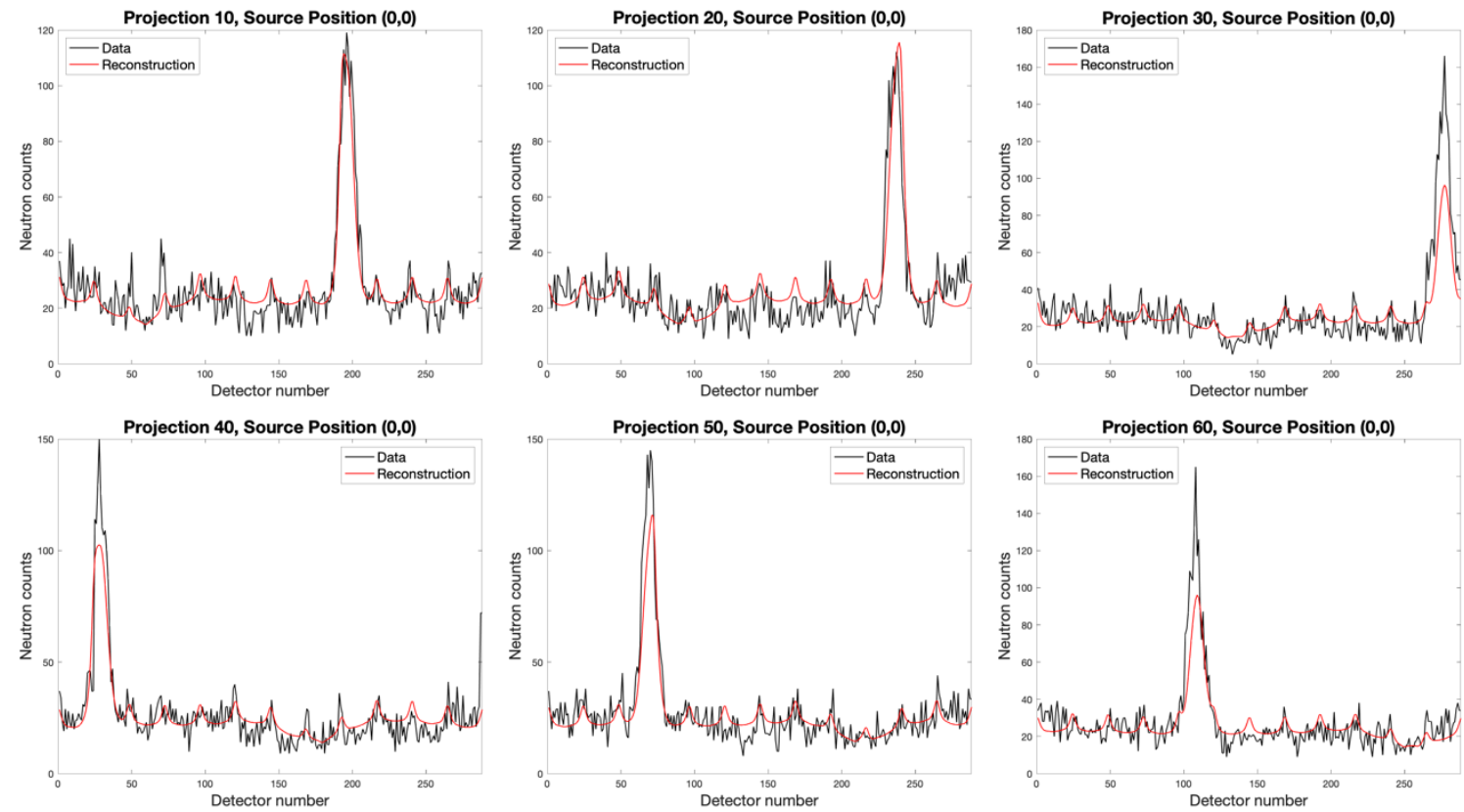

Figure 15. Measured data compared to reconstruction for position $(0,0)$, projections 10, 20, 30, 40, 50, and 60 .

The reconstructed image corresponding to the reconstructed sinogram is shown in Figure 16 for (left) the entire $35 \mathrm{~cm} \times 35 \mathrm{~cm}$ field of view and (right) for a zoomed $12 \mathrm{~cm} \times 12 \mathrm{~cm}$ region. The apparent size of the reconstructed source is approximately $4 \mathrm{~mm}$ FWHM, similar to the true source size which resides within a $6 \mathrm{~mm}$ capsule. The location of the most intense pixel in the reconstructed image corresponds to position $(0,0)$, which indicates that the fixture has been aligned well with the imager. The integrated intensity of the source from the reconstructed image is $3.05 \times 10^{6}$. This number of neutrons is only $71.6 \%$ of the emitted neutrons per projection $\left(4.26 \times 10^{6}\right)$, but careful calibration of the imager has not yet been performed. 

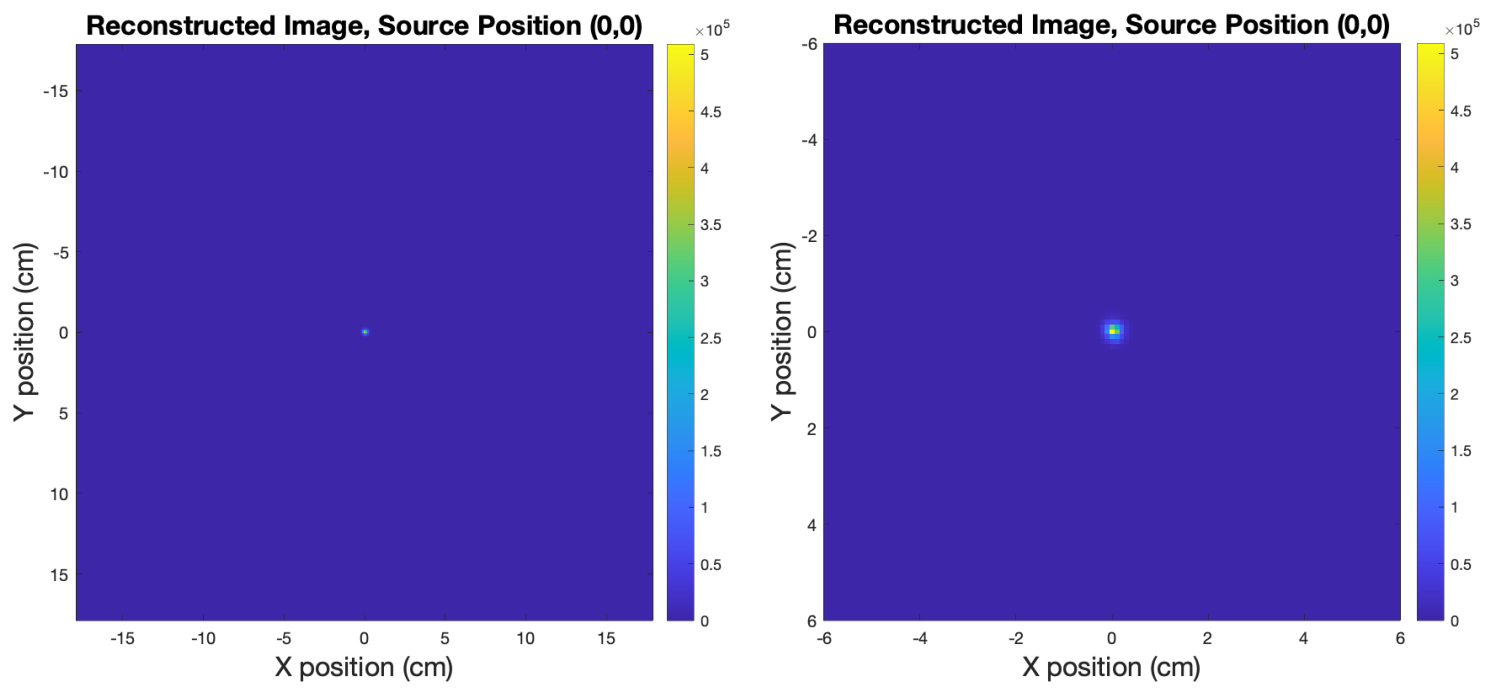

Figure 16. The reconstructed image for position $(0,0)$ for (left) the entire field of view and (right) a zoomed region $12 \mathrm{~cm} \times 12 \mathrm{~cm}$.

\subsubsection{Position $(0,4)$}

The (left) measured and (right) reconstructed sinograms for the source positioned at $(0,0)$ are shown in Figure 17. The data were reconstructed using the MLEM algorithm for 1,000 iterations.
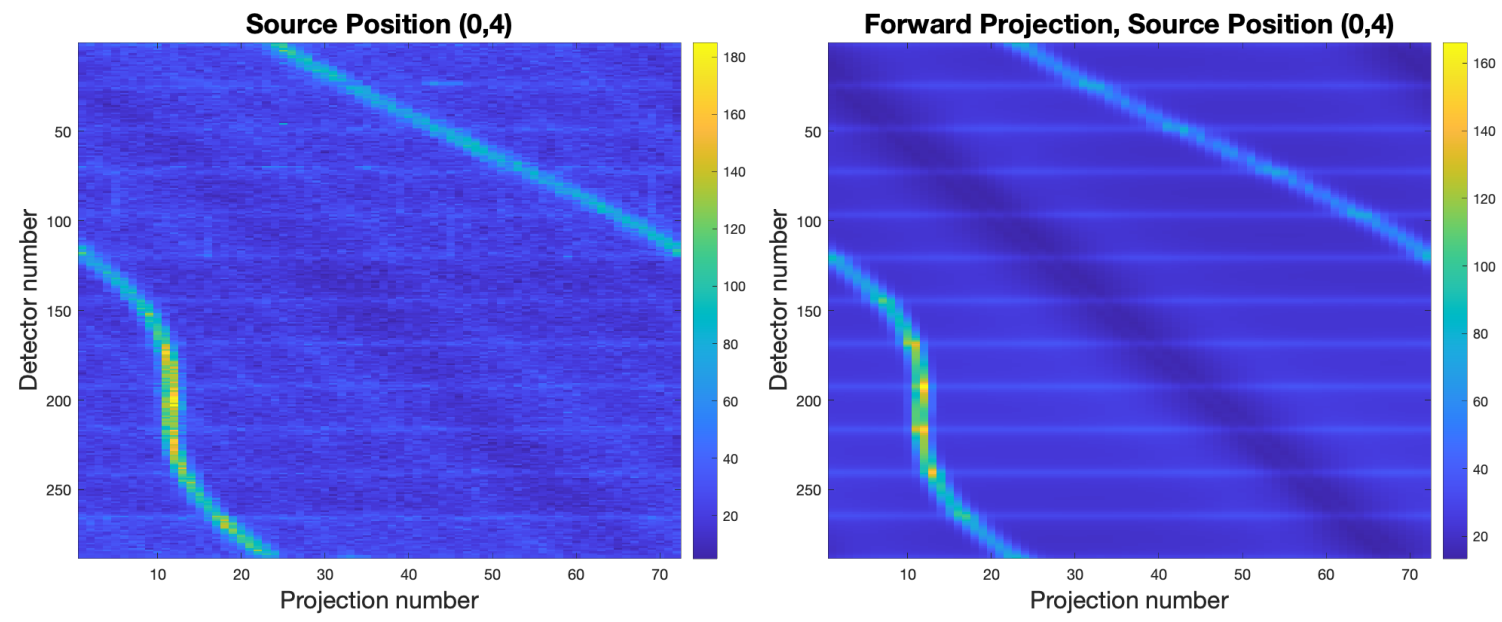

Figure 17: The (left) measured and (right) reconstructed sinogram for source position $(0,4)$

Visual comparison of the measured and reconstructed sinograms in Figure 17 enables assessment to determine whether sinogram features are reproduced by the reconstruction, but quantitative assessment is more easily accomplished in one-dimensional plots. As a result, the (black) measured and (red) reconstructed detector counts for projection numbers 10, 20, 30, 40, 50, and 60 are shown in Figure 18. Note that the peak counts are well reproduced in projection 10 but appear roughly $50 \%$ off in the remainder of the projections. As indicated earlier, efficiency calibration of both the detectors and the individual slits will be a top priority of future characterization of the as-built imager. 

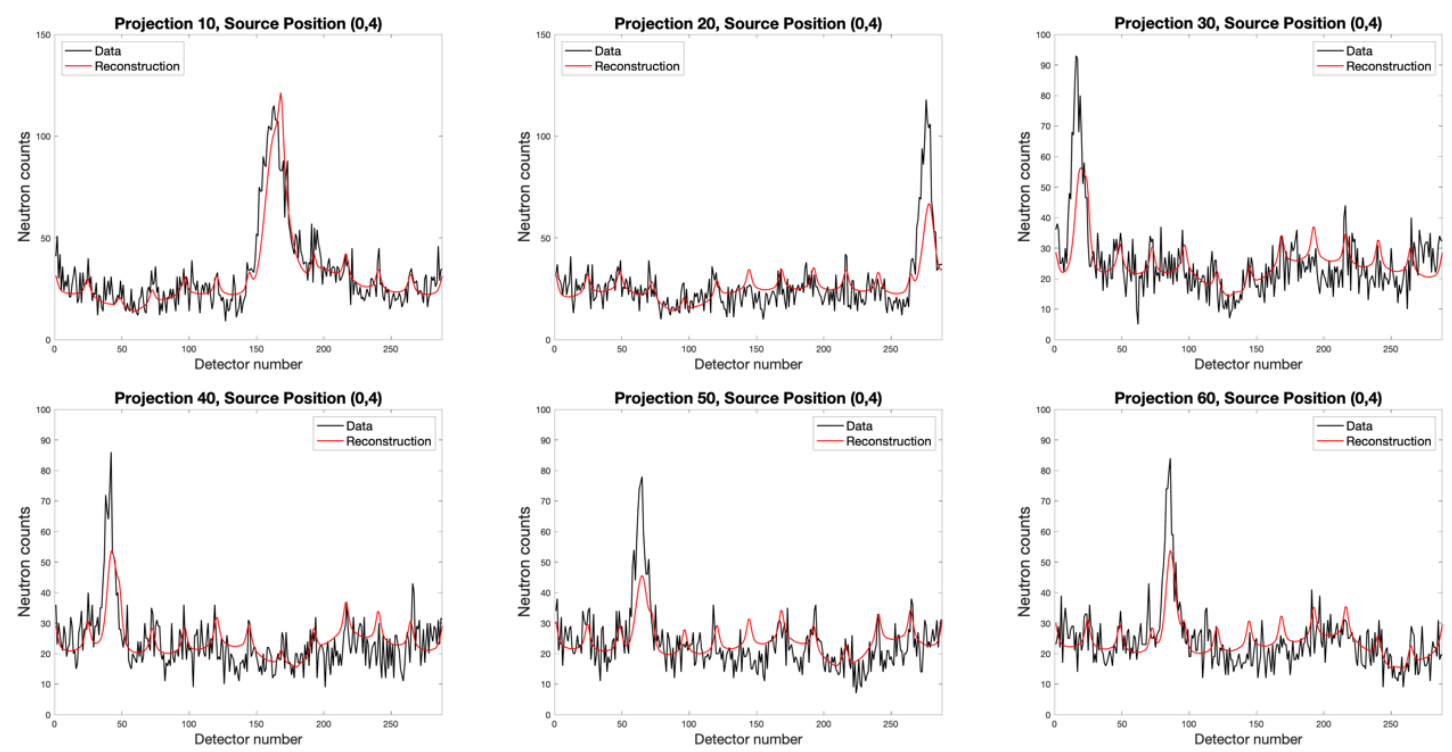

Figure 18: Measured data compared to reconstruction for position $(0,4)$, projections 10, 20, 30, 40, 50, and 60 .

The reconstructed image corresponding to the reconstructed sinogram is shown in Figure 19 for a zoomed $12 \mathrm{~cm} \times 12 \mathrm{~cm}$ region. The apparent size of the reconstructed source is approximately $5 \mathrm{~mm}$ FWHM, slightly larger than the reconstructed size at position $(0,0)$ but still similar to the true source size which resides within a $6 \mathrm{~mm}$ capsule. The position of the most intense pixel of the reconstructed image is $(-1.705,-4.513)$, or $4.82 \mathrm{~cm}$ from the origin. This distance is $95 \%$ of the expected $5.08 \mathrm{~cm}$, so the origin of the length scale difference will need to be investigated. The integrated intensity of the source from the reconstructed image is $3.15 \times 10^{6}$ emitted neutrons. This value is $72.1 \%$ of the emitted neutrons per projection $\left(4.37 \times 10^{6}\right)$, so while careful calibration of the imager still needs to be performed, the reconstructed values for the same source at the two positions agree to within a percent. This repeatability is encouraging.

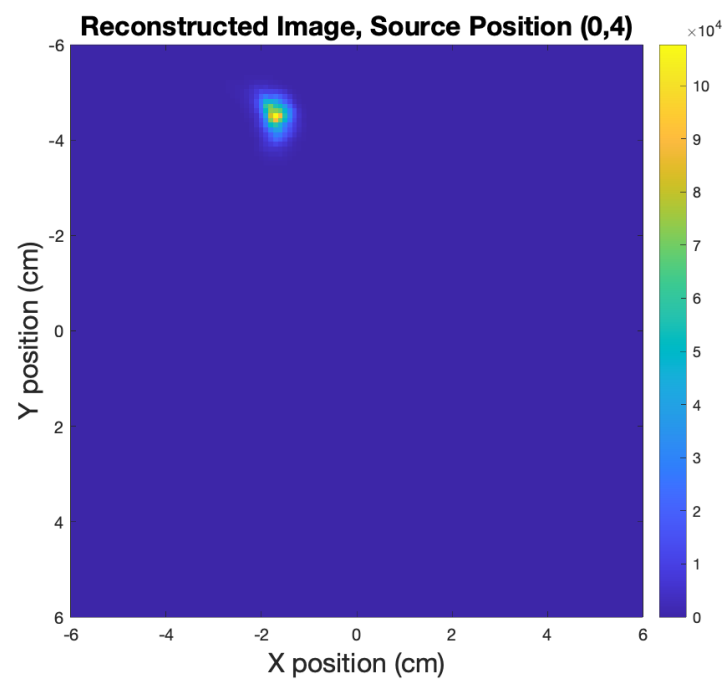

Figure 19: The reconstructed image of position $(0,4)$ for a zoomed region $12 \mathrm{~cm} \times 12 \mathrm{~cm}$. 


\subsubsection{Position (2,0)}

The (left) measured and (right) reconstructed sinograms for the source positioned at $(2,0)$ are shown in Figure 20. The data were reconstructed using the MLEM algorithm for 1,000 iterations.
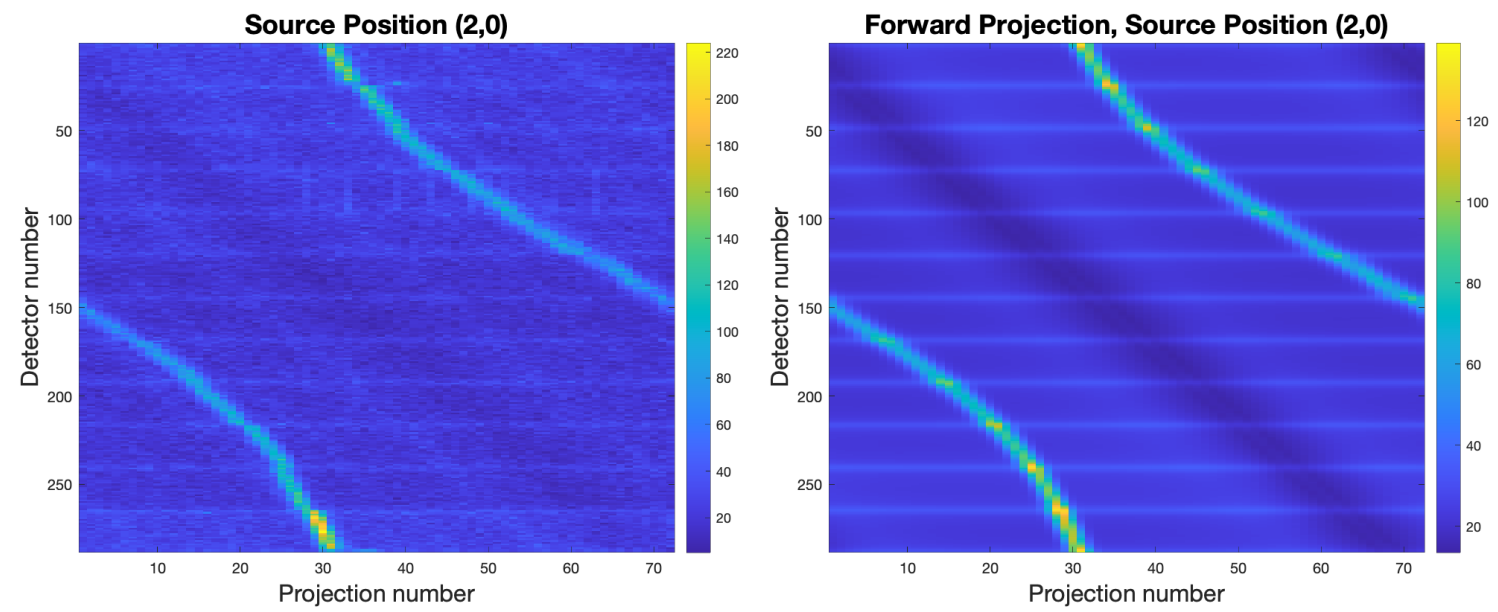

Figure 20: The (left) measured and (right) reconstructed sinogram for source position $(2,0)$

As before, visual comparison of the measured and reconstructed sinograms in Figure 20 enables assessment to determine whether sinogram features are reproduced by the reconstruction, but quantitative assessment is more easily accomplished in one-dimensional plots. As a result, the (black) measured and (red) reconstructed detector counts for projection numbers 10, 20, 30, 40, 50, and 60 are shown in Figure 21. Note that the peak counts are well reproduced in projection 20,40, and 60 but appears as much as $50 \%$ off in projection 30 . As indicated earlier, efficiency calibration of both the detectors and the individual slits will be a top priority of future characterization of the as-built imager.
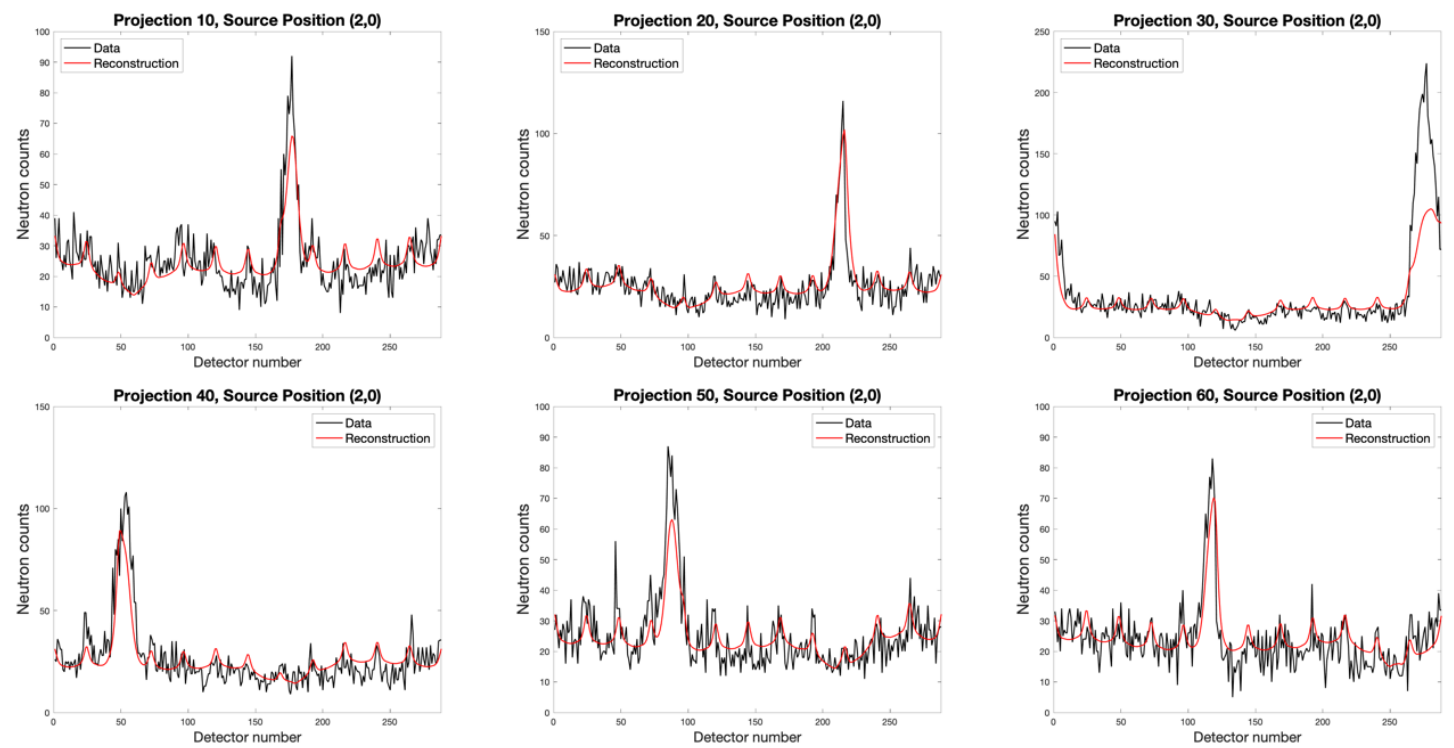

Figure 21: Measured data compared to reconstruction for position $(2,0)$, projections 10, 20, 30, 40, 50, and 60 . 
The reconstructed image corresponding to the reconstructed sinogram is shown in Figure 22 for a zoomed $12 \mathrm{~cm} \times 12 \mathrm{~cm}$ region. The apparent size of the reconstructed source is approximately $5 \mathrm{~mm}$ FWHM, slightly larger than the reconstructed size at position $(0,0)$ but still similar to the true source size which resides within a $6 \mathrm{~mm}$ capsule. The position of the most intense pixel of the reconstructed image is $(-2.407,-0.903)$, or $2.57 \mathrm{~cm}$ from the origin. In contrast to the previous measurement, this distance is nearly exactly the expected $2.54 \mathrm{~cm}$. However, the origin of the length scale difference between this measurement and the previous needs to be investigated. In particular, it is likely that the mechanical robustness of the fixture needs to be improved to ensure repeatable positioning. The integrated intensity of the source from the reconstructed image is $3.09 \times 10^{6}$ emitted neutrons. This value is $72.7 \%$ of the emitted neutrons per projection $\left(4.25 \times 10^{6}\right)$, so while careful calibration of the imager still needs to be performed, the reconstructed values for the same source at the various positions have good agreement.

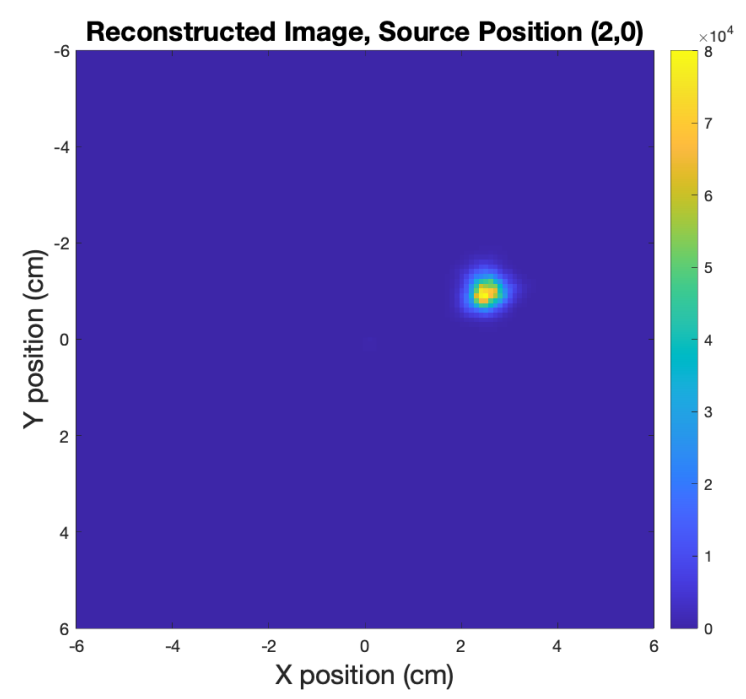

Figure 22: The reconstructed image of position $(2,0)$ for a zoomed region $12 \mathrm{~cm} \times 12 \mathrm{~cm}$.

\subsection{RECONSTRUCTION OF FIVE-SOURCE MEASUREMENT}

The data from the individual measurements of positions $(0,0),(0,4),(2,0),(1,3)$, and $(2,1)$ were summed to create a measurement of five sources. The positions were chosen so that pairs of sources were positioned distances of $1, \sqrt{2}, 2,5$, and 4 times the closest possible spacing in the assembly. The resulting measured neutron counts in each detector for each rotation angle is plotted on the left of Figure 23, and the corresponding reconstructed values on the right of Figure 23. A background measurement with no sources present was subtracted from each of the individual measurements, so the measured counts distributed across all detectors are associated with the presence of the source. These counts reach the detectors either by penetrating the collimator or leaving the collimator bore by the top or bottom and reflecting back to the detectors as room return. 

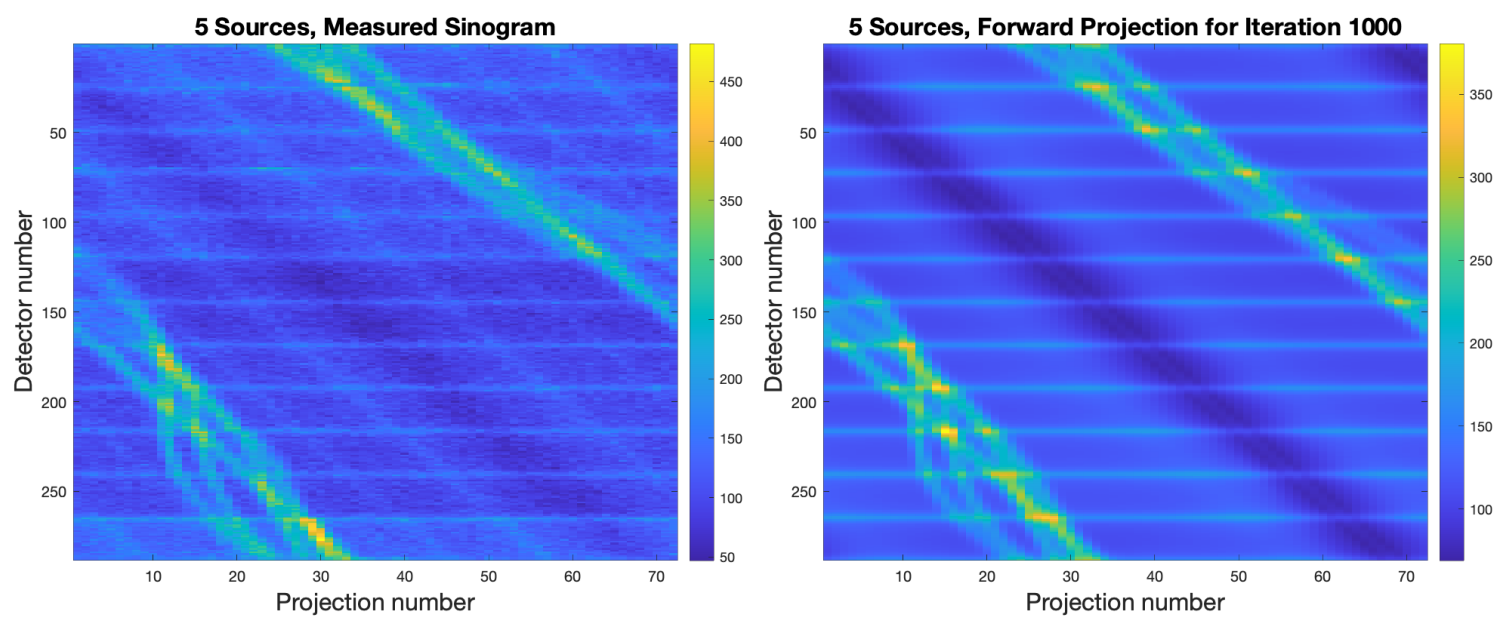

Figure 23. The (left) measured and (right) reconstructed sinograms for the sum of five positions.

The data were reconstructed using the MLEM algorithm for 1,000 iterations. The reconstruction reproduces the visual features of the simulated data. To aid in comparison between the simulated measurement and reconstruction, comparison for projections 10, 20, 30, 40, 50, and 60 are shown in Figure 24.
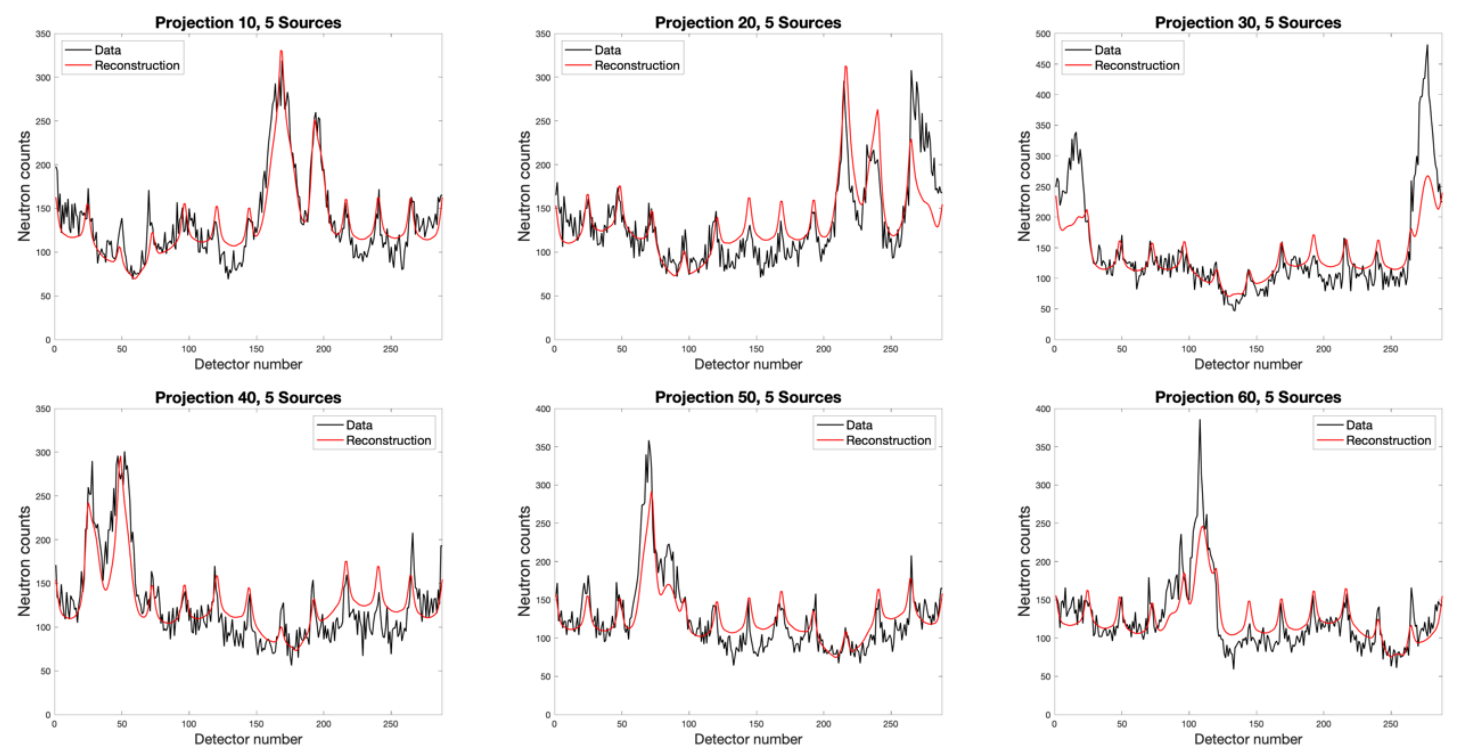

Figure 24. Comparison of measurement and MLEM reconstruction for projections 10, 20, 30, 40, 50, and 60.

The reconstructed image that corresponds to the sinogram is shown in Figure 25 for (left) 100 iterations and (right) 1000 iterations to illustrate the slow convergence of the algorithm. The different source slots are labeled on the 1000-iteration image. In this combined image, the integrated intensity of the source at position $(0,0)$ is $3.85 \times 10^{6}$ emitted neutrons while the integrated intensity of the source at position $(0,4)$ is $2.28 \times 10^{6}$ emitted neutrons, only $59 \%$ of the former. In the image, the total intensity is correct, but its apportionment between positions is not correct. The expectation of the authors is that careful calibration of the response will rectify this situation. 

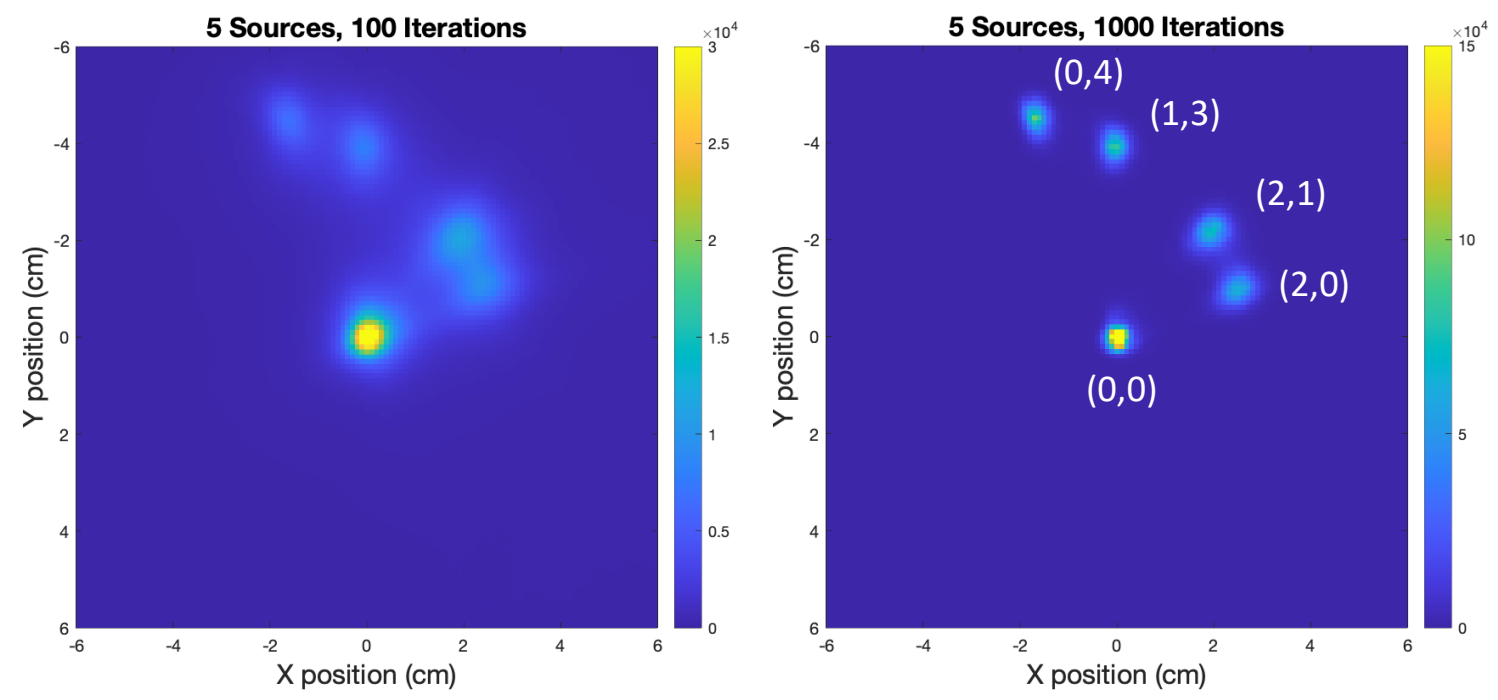

Figure 25. Image of five sources reconstructed using the MLEM algorithm for (left) 100 iterations and (right) 1000 iterations.

Note that each of the sources is resolved from the others including the sources spaced at the closest distance from each other. This result indicates that, combined with iterative reconstruction, the imager is expected to have sufficient spatial resolution to resolve individual fuel pins. Also note that because the system matrix is not sparse, large numbers of iterations are needed to resolve sources close to one another.

\section{SUMMARY AND OUTLOOK}

The present work continues development of the parallel-slit ring collimator for passive neutron emission imaging of spent fuel through commissioning and first measurements with the laboratory prototype imager. The results of these initial measurements indicate that resolution of neighboring fuel rods in a nuclear fuel assembly is possible. Although initial results are promising, substantial work remains to demonstrate quantitative imaging of neutron sources across the field of view. Elements of this work are enumerated below.

At present, radiation transport simulations of the imager were built for a 96-slit configuration but need to be modified to reflect as-built dimensions and 72 slits. These simulations will be used to help calibrate the imager response and to tune the analytical model for the imager response so that it matches for the outer radius of the inspection volume as well as to perform simulations of tomographic measurements of spent fuel assemblies. Measurement will be aided by including more information in the data stream, including additional signals to monitor live time and collimator position. As a result, a pulser input will be added to monitor for live time and optical switches will be added to monitor slit edges and full rotation of the collimator. Additional calibration of the imager is also needed, including measurement of the relative efficiency of the detectors and of the slits. The relative detector efficiency will be calibrated using a neutron source affixed to the collimator as it rotates to illuminate each detector with equal neutrons in turn. The slit efficiency will be measured by affixing the source to the fixture near the edge of the field of view so that each slit will be illuminated with equal neutrons in turn. The rigidity of the source fixture will likely need to be improved in order to ensure that there cannot be relative motion of the top plate with respect to the bottom plate during movement of the source rod. Additional characterization will also be needed to understand the increased count rate observed at the detector edges, which may require replacing the HDPE on the sides of the detector with borated polyethylene. Perhaps most importantly, tomographic 
data needs to be acquired for a neutron source in each position of the source fixture with and without stainless-steel shielding rods in place. Last of all, the gamma-ray insensitivity of the as-built neutron detectors should be verified, which may require dismounting a detector to bring it to a facility where it can be tested with an appropriately large radiation field.

\section{ACKNOWLEDGEMENTS}

This work is supported by the US Department of Energy, Office of Defense Nuclear Nonproliferation Research and Development in the National Nuclear Security Administration (NA-22). This material is based in part upon work supported by the Department of Energy National Nuclear Security Administration through the Nuclear Science and Security Consortium under Award Number DENA0003180.

\section{REFERENCES}

[1] T. Honkamaa et al., “A Prototype for Passive Gamma Emission Tomography,” International Atomic Energy Agency Safeguards Symposium, 2014.

[2] M. Mayorov et al., "Gamma Emission Tomography for the Inspection of Spent Nuclear Fuel," 2017 IEEE Nuclear Science Symposium and Medical Imaging Conference (NSS/MIC), Atlanta, GA, 2017, pp. 1-2.

[3] P. A. Hausladen, A. S. Iyengar, L. Fabris, J. Yang, and J. Hu, "A Design Study of the Parallel-Slit Ring Collimator for Fast Neutron Emission Tomography of Spent Fuel," Report no. ORNL/SPR2018/975, Oak Ridge National Laboratory, Oak Ridge, TN, 2018.

[4] Iyengar, Anagha Srikanth, "The Design of an Imager to Safeguard Spent Fuel Using Passive Fast Neutron Emission Tomography. " PhD diss., University of Tennessee, 2019. https://race.tennessee.edu/utk_graddiss/5343

[5] P. A. Hausladen, M. E. Montague, A. S. Iyengar, J. Yang, "Tomographic Image Reconstruction for the Parallel-Slit Ring Collimator Fast Neutron Emission Tomography System," Report no. ORNL/SPR-2020/1510, Oak Ridge National Laboratory, Oak Ridge, TN, 2020.

[6] A. Iyengar, P. Hausladen, J. Yang, L. Fabris, J. Hu. J. Lacy, A. Athanasiades, "Detection of fuel pin diversion via fast neutron emission tomography", in $39^{\text {th }}$ ESARDA Annual Meeting-Symposium, 16-18 May 2017, Dusseldorf, Germany.

[7] P. A. Hausladen, A. S. Iyengar, L. Fabris, J. Yang, J. Hu, and A. L. Lousteau, "Modelling Passive Fast Neutron Emission Tomography of Spent Nuclear Fuel", in Tagziria, H., Bourva, L., Jansson, P.; International Workshop on Numerical Modelling of NDA Instruments and Methods for Nuclear Safeguards; Luxembourg: Publications Office of the European Union, 2018; ISBN 978-92-7998443-3; doi:10.2760/055930; JRC114178.

[8] A. S. Iyengar, P. A. Hausladen, L. Fabris, J. Yang, and J. Hu, "Development of a Nuclear Fuel Safeguards Verification Technology for New Facility Types: Use of Fast Neutron Emission Tomography for Spent Fuel Verification”, IAEA Symposium on International Safeguards, Vienna, Austria, November 2018 